\title{
OPEN Enhanced firing of locus coeruleus neurons and SK channel dysfunction are conserved in distinct models of prodromal Parkinson's disease
}

\author{
Lina A. Matschke ${ }^{1,2,6}$, Marlene A. Komadowski ${ }^{1,6}$, Annette Stöhr ${ }^{1}$, Bolam Lee ${ }^{2}$, \\ Martin T. Henrich ${ }^{2}$, Markus Griesbach ${ }^{1}$, Susanne Rinné ${ }^{1}$, Fanni F. Geibl2 ${ }^{2}$, Wei-Hua Chiu ${ }^{2}$, \\ James B. Koprich ${ }^{3}$, Jonathan M. Brotchie ${ }^{3}$, Aytug K. Kiper ${ }^{1}$, Amalia M. Dolga ${ }^{4}$, \\ Wolfgang H. Oertel ${ }^{2,5,7}$ \& Niels Decher ${ }^{1,7}$,
}

Parkinson's disease (PD) is clinically defined by the presence of the cardinal motor symptoms, which are associated with a loss of dopaminergic nigrostriatal neurons in the substantia nigra pars compacta (SNpc). While SNpc neurons serve as the prototypical cell-type to study cellular vulnerability in $P D$, there is an unmet need to extent our efforts to other neurons at risk. The noradrenergic locus coeruleus (LC) represents one of the first brain structures affected in Parkinson's disease (PD) and plays not only a crucial role for the evolving non-motor symptomatology, but it is also believed to contribute to disease progression by efferent noradrenergic deficiency. Therefore, we sought to characterize the electrophysiological properties of LC neurons in two distinct PD models: (1) in an in vivo mouse model of focal $\alpha$-synuclein overexpression; and (2) in an in vitro rotenone-induced PD model. Despite the fundamental differences of these two PD models, $\alpha$-synuclein overexpression as well as rotenone exposure led to an accelerated autonomous pacemaker frequency of $L C$ neurons, accompanied by severe alterations of the afterhyperpolarization amplitude. On the mechanistic side, we suggest that $\mathrm{Ca}^{2+}$-activated $\mathrm{K}^{+}(\mathrm{SK})$ channels are mediators of the increased LC neuronal excitability, as pharmacological activation of these channels is sufficient to prevent increased LC pacemaking and subsequent neuronal loss in the LC following in vitro rotenone exposure. These findings suggest a role of SK channels in PD by linking $\alpha$-synuclein- and rotenone-induced changes in LC firing rate to SK channel dysfunction.

Parkinson's disease (PD), the second most common neurodegenerative disorder, is a progressive, age-related movement disorder. It is characterized by the accumulation of $\alpha$-synuclein ( $\alpha$ Syn) positive eosinophilic neuronal inclusions, termed Lewy pathology, and neurodegeneration. Importantly, even in late stage PD patients, Lewy pathology and neuronal cell loss are confined to certain vulnerable brain regions ${ }^{1,2}$. Affected neurons share a set of morphological and functional traits going along with a high energetic and metabolic demand. These traits include e.g. long and highly branched axons, a large number of neurotransmitter release sites, and slow tonic pacemaking activity ${ }^{2}$. While dopaminergic substantia nigra (SN) neurons serve as the prototypical cell-type to study cellular vulnerability in PD, there is an unmet need to extend our efforts to study other neurons at risk. The

\footnotetext{
${ }^{1}$ Institute for Physiology and Pathophysiology, Vegetative Physiology and Marburg Center for Mind, Brain and Behavior - MCMBB, Philipps-University Marburg, 35037 Marburg, Germany. ${ }^{2}$ Clinic for Neurology, Philipps-University Marburg, 35043 Marburg, Germany. ${ }^{3}$ Krembil Research Institute, Toronto Western Hospital, University Health Network, 8KD402, Toronto, ON M5T 2S8, Canada. ${ }^{4}$ Faculty of Science and Engineering, Groningen Research Institute of Pharmacy, Department of Molecular Pharmacology, University of Groningen, 9713 AV Groningen, The Netherlands. ${ }^{5}$ Hertie Senior Research Professor of the Charitable Hertie Foundation, 60323 Frankfurt am Main, Germany. ${ }^{6}$ These authors contributed equally: Lina A. Matschke and Marlene A. Komadowski. ${ }^{7}$ These authors jointly supervised this work: Wolfgang $\mathrm{H}$. Oertel and Niels Decher. ${ }^{\boxplus}$ email: decher@staff.uni-marburg.de
} 
noradrenergic locus coeruleus (LC), a small nucleus in the pontine brainstem which constitutes the major source of noradrenaline (NA) in the central nervous system, has been identified as a key structure in the prodromal or premotor stage of $\mathrm{PD}^{3,4}$. Results from previous studies show that LC cells suffer from aSyn aggregation and Lewy pathology formation several years if not decades before the degeneration of dopaminergic SN neurons ${ }^{5,6}$. Further, LC cell loss might even exceed nigral neurodegeneration ${ }^{7}$. LC dysfunction and neurodegeneration are associated with several non-motor symptoms of PD, such as anxiety, depression or reduced arousal ${ }^{8,9}$. Additionally, noradrenaline released from LC efferent neurons has been shown to exert potent anti-inflammatory effects $^{10,11}$. Given the central role of neuroinflammation in neurodegenerative disorders ${ }^{12}$, it is not surprising that LC ablation has been shown to aggravate nigral neurodegeneration in toxin models of $\mathrm{PD}^{13-15}$, whereas genetic or pharmacological enhancement of noradrenergic neurotransmission was able to mitigate midbrain pathology and PD symptoms ${ }^{11,16,17}$. Hence, affection of the LC-noradrenergic system during PD has been proposed to not only contribute to evolving non-motor symptomatology, but due to efferent noradrenergic deficiency and the absent anti-inflammatory effect also to disease progression. Thus, studies aimed at investigating LC neuronal (dys)function prior to marked neurodegeneration may not only provide important insights into the mechanisms leading to noradrenergic imbalance and LC cell loss, but foster identification of potential cellular targets for disease modifying therapy approaches.

Therefore, we sought to characterize the electrophysiological alterations of LC neurons at the single cell level in two distinct PD models. To this end, we conducted whole-cell patch clamp experiments of LC neurons in: (1) a well characterized in vivo mouse model relying on viral vector mediated focal overexpression of human wild-type (WT) or human A53T-mutated $\alpha \mathrm{Syn}^{18}$; and (2) in an in vitro PD model based on the application of the mitochondrial complex I inhibitor rotenone. Our results yielded three key observations: First, despite the fundamental differences between these two models, aSyn overexpression as well as rotenone exposure led to a significantly accelerated autonomous LC pacemaker frequency which was associated with a marked decrease of the afterhyperpolarization amplitude. Second, we identified small-conductance $\mathrm{Ca}^{2+}$-activated $\mathrm{K}^{+}$(SK) channels as mediators of the significantly increased excitability of LC neurons in both models. Third, our experiments suggest that SK channels might represent valuable pharmacological targets for disease modifying therapy approaches, since application of the SK channel activator, 6,7-dichloro-1H-indole-2,3-dione 3-oxime (NS309) not only normalized LC firing frequency, but even counteracted neuronal LC loss in the in vitro rotenone model. These novel findings raise the possibility that SK channels are possible mediators of LC neurodegeneration in PD.

\section{Results}

Progressive acceleration of the pacemaker frequency after overexpression of human mutated $\alpha S y n^{A 53 T}$ in LC neurons. To study whether and in which time-frame overexpression of aSyn alters the firing behavior of LC neurons, recombinant adeno-associated viral (rAAV) vectors containing the gene for either human wild-type $\alpha \mathrm{Syn}\left(\mathrm{AAV}-\alpha \mathrm{Syn}^{\mathrm{WT}}\right)$, human mutant $\alpha \mathrm{Syn}\left(\mathrm{AAV}-\alpha \mathrm{Syn}^{\mathrm{A} 53 \mathrm{~T}}\right)$ or luciferase as a control (AAVluc) (Fig. 1a) were injected into the right $\mathrm{LC}$ of $\mathrm{C} 57 \mathrm{BL} / 6 \mathrm{~N}$ wild-type mice. In a recent study, we already confirmed that these vectors lead to equal transduction rates of LC neurons ${ }^{18}$. Mice were consecutively sacrificed 1, 3, 6 and 9 weeks post-injection and electrophysiological recordings of LC neurons were performed in acute brainstem slices (Fig. 1b). To verify the noradrenergic nature of the investigated neurons and probe that they express the injected proteins, neurons were filled with NB via the pipette during patch clamp recording and post hoc co-stained for TH, the rate limiting enzyme in noradrenaline synthesis, and human aSyn (haSyn) (Fig. 1c).

In a first set of experiments, spike trains in the whole-cell current clamp configuration were recorded to elucidate alterations of the spontaneous pacemaker activity of LC neurons. Representative recordings of spontaneously active luc, $\alpha S_{y n}{ }^{\mathrm{WT}}$ and $\alpha \mathrm{Syn}^{\mathrm{A} 53 \mathrm{~T}}$ overexpressing LC neurons are displayed in Fig. 1d for each time-point. At one week post-injection, each group showed spontaneous pacemaker activity with frequencies of $\sim 3 \mathrm{~Hz}$, which corresponds to the pacemaker frequency described for non-manipulated LC neurons ${ }^{19-21}$. However, over the time-frame of 9 weeks, a gradual acceleration of firing frequency was detected in $\alpha \mathrm{Syn}^{\mathrm{A} 53 \mathrm{~T}}$, but not in luc overexpressing LC neurons. Note that for $\alpha_{S y n}{ }^{W T}$ overexpressing neurons there was also a tendency towards increased firing rates after 9 weeks compared to luc injected animals which did however not reach statistical significance (Fig. le and Supplementary Fig. S1). Notably, after 9 weeks, the mean pacemaker frequency of $\alpha \mathrm{Syn}^{\mathrm{A} 53 \mathrm{~T}}$ overexpressing neurons was approximately two-fold higher than that of luc control (Fig. 1e and Supplementary Fig. S1). In order to analyze the precision of pacemaking, the coefficient of variation (CV) was measured as previously described ${ }^{22,23}$. Under control conditions, the CV of LC neurons was $\sim 18 \%$, reflecting high precision of the pacemaker mechanism. $\alpha \mathrm{Syn}^{\mathrm{WT}}$ or $\mathrm{SSyn}^{\mathrm{A} 53 \mathrm{~T}}$ did not alter the precision of pacemaking in LC neurons, since the $\mathrm{CV}$ was not significantly changed (Fig. 1f). However, we observed clear time-dependent alterations of the action potential (AP) waveform of $\alpha \mathrm{Syn}^{\mathrm{A} 53 \mathrm{~T}}$ and $\alpha \mathrm{Syn}^{\mathrm{WT}}$ overexpressing neurons (Fig. $1 \mathrm{~g}$ ). Compared to luc controls, the afterhyperpolarization (AHP) amplitude was significantly reduced in $\alpha \mathrm{Syn}^{\mathrm{A} 53 \mathrm{~T}}$ overexpressing LC neurons at 6 and 9 weeks post-injection, and in $\alpha \mathrm{Syn}^{\mathrm{WT}}$ overexpressing mice at 9 weeks post-injection (Fig. 1h). In contrast, $\mathrm{AP}$ duration at $50 \%$ of the peak $\left(\mathrm{APD}_{50}\right)$ and $\mathrm{AP}$ threshold remained unchanged (Fig. 1i,j). In summary, our data show that overexpression of $\alpha \mathrm{Syn}^{\mathrm{A} 53 \mathrm{~T}}$ led to a time-dependent amplification of the LC neurons' excitability.

$\alpha$ Syn induced, progressive alterations of $\mathrm{Ca}^{2+}$ - and small conductance $\mathrm{Ca}^{2+}$-activated $\mathrm{K}^{+}$currents in LC neurons. To investigate the underlying biophysical mechanisms of aSyn-induced increase of LC neuron firing, we analyzed whether distinct pacemaker currents were altered in these models. We have previously described that L- and T-type voltage-dependent $\mathrm{Ca}^{2+}(\mathrm{Cav})$ channels, as well as SK channels play important roles in the regulation of LC pacemaker frequency and shape of the AHP ${ }^{21,22}$. In addition, A-type and delayed rectifier $\mathrm{K}^{+}$channels are known to be functionally expressed in LC neurons ${ }^{24,25}$. Therefore, we performed wholecell voltage clamp recordings utilizing different voltage protocols to analyze $\mathrm{K}^{+}, \mathrm{Ca}^{2+}$, and $\mathrm{Ca}^{2+}$ activated $\mathrm{K}^{+}$cur- 

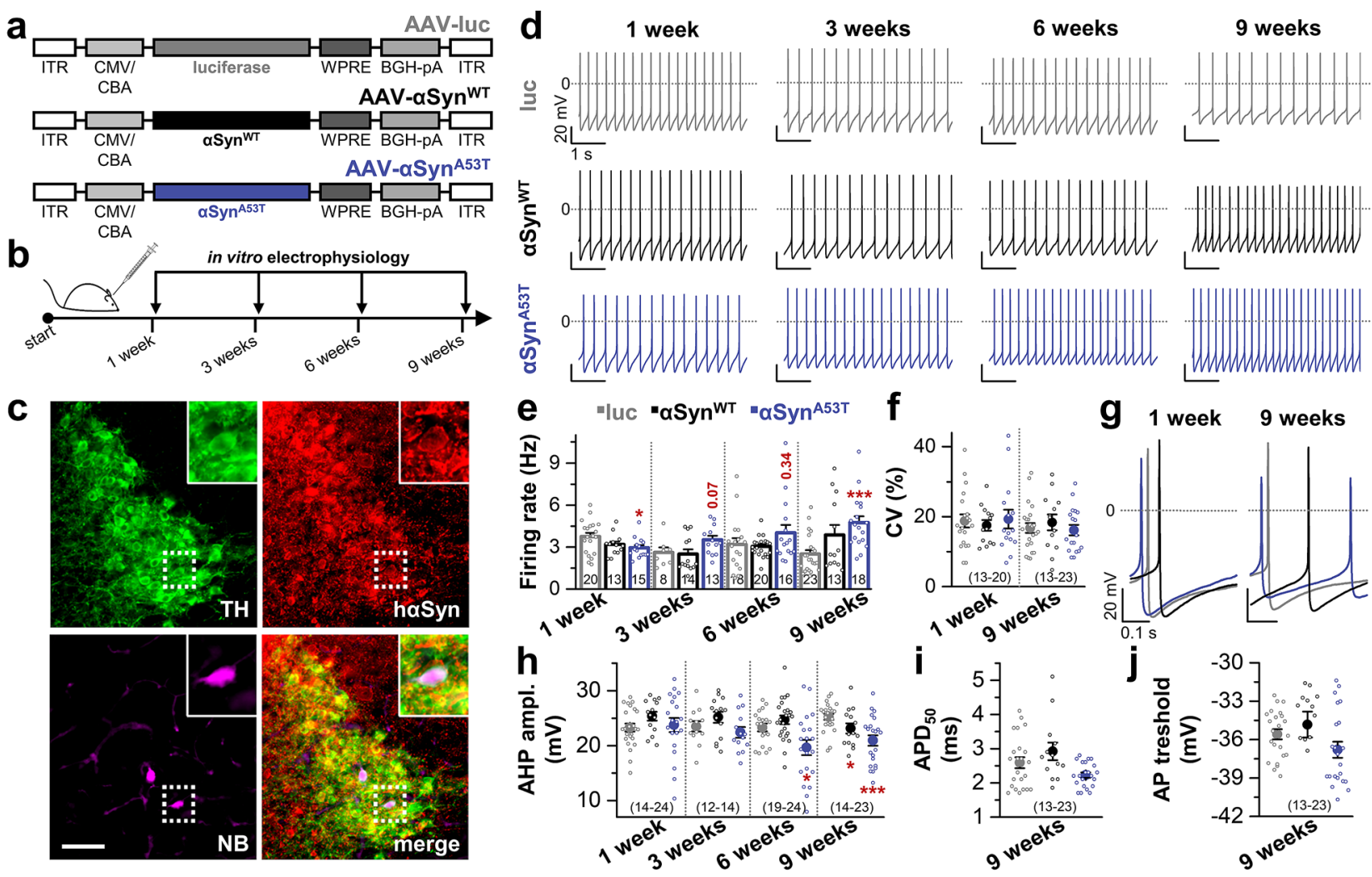

Figure 1. rAAV mediated $\alpha S n^{\mathrm{A} 53 \mathrm{~T}}$ overexpression induces acceleration of the intrinsic pacemaker frequency in LC neurons. (a) rAAV1/2 vectors containing a chicken $\beta$-actin promoter hybridized with a CMV immediate early enhancer sequence (CMV/CBA) were injected into the right LC to drive expression of $\alpha \mathrm{Syn}^{\mathrm{WT}}, \alpha \mathrm{Syn}^{\mathrm{A}}{ }^{53 \mathrm{~T}}$, or luc (control). WPRE, woodchuck hepatitis virus posttranscriptional regulatory element; BGH-pA, bovine growth hormone polyadenylation sequence; ITR, inverted terminal repeat. (b) Scheme illustrating the experimental design of the study. Mice were consecutively sacrificed 1, 3, 6 and 9 weeks after injection of rAAV vectors for in vitro electrophysiology. (c) Representative triple immunofluorescence staining for TH (green), human $\alpha$ Syn (red), or neurobiotin (NB, magenta). Co-localization identifies patched, NB-filled neurons as THand $\alpha$ Syn-positive. Scale bar: $100 \mu \mathrm{m}$. (d) Example recordings of spontaneously active luc, $\alpha \mathrm{Syn}^{\mathrm{WT}}$ or $^{\mathrm{aSyn}}{ }^{\mathrm{A} 53 \mathrm{~T}}$ overexpressing LC neurons in the whole-cell current clamp configuration at 1,3,6 and 9 weeks after injection in the presence of synaptic blockers. (e) Quantification of the pacemaker frequency revealed a significant acceleration of action potential firing 9 weeks post-injection in $a S^{A} n^{A 53 T}$ overexpressing animals compared to luc controls. (f) Analysis of the CV as a measure for regularity of the data shown in (e). (g) Representative AP of luc (grey), $\alpha$ Syn $^{\mathrm{WT}}$ (black) and ${ }^{\mathrm{WSyn}}{ }^{\mathrm{A} 53 \mathrm{~T}}$ (blue) overexpressing animals 1 and 9 weeks post-injection. The $\mathrm{AP}$ waveform of $\alpha \mathrm{Syn}^{\mathrm{A} 53 \mathrm{~T}}$ overexpressing neurons showed time-dependent alterations. $(\mathbf{h}-\mathbf{j})$ Analyses of basic AP properties revealed a time-dependent reduction of the AHP amplitude in $\alpha S_{y n}{ }^{\mathrm{WT}}$ overexpressing mice at 9 weeks post-injection and in $\alpha \mathrm{Syn}^{\mathrm{A}}{ }^{\mathrm{5} 3 \mathrm{~T}}$ overexpressing mice at 6 and 9 weeks post-injection (h), whereas $\mathrm{APD}_{50}$ (i) and AP threshold (j) remained unchanged. Data are represented as mean \pm SEM and also as individual data points. ${ }^{\star} p<0.05$; ${ }^{* * *} p<0.001$. [(e) 1 week: unpaired Student's $t$-test vs. luc; 3 weeks: $\alpha$ Syn ${ }^{\text {WT }}$, Mann-Whitney-U test vs. luc/aSyn ${ }^{\mathrm{A} 53 \mathrm{~T}}$, unpaired Student's $t$-test vs. luc; 6 weeks: Mann-Whitney-U test vs. luc; 9 weeks: $\alpha_{\text {Syn }}{ }^{\text {TT }}$, Mood's median test vs. luc/aSyn ${ }^{\mathrm{A} 53 \mathrm{~T}}$, Mann-Whitney-U test vs. luc. (f) unpaired Student's $t$-test vs. corresponding luc. (h) 1 week and 3 weeks: unpaired Student's $t$-test vs. corresponding luc; 6 and 9 weeks: $\alpha \operatorname{Syn}^{\mathrm{WT}}$, unpaired Student's $t$-test vs. corresponding luc/aSyn ${ }^{\mathrm{A} 53 \mathrm{~T}}$, Welch's $t$-test vs. corresponding luc. (i,j) Mann-Whitney-U test vs. corresponding luc].

rents at each time-point following overexpression of luc, $\alpha \mathrm{Syn}^{\mathrm{WT}}$ and $\alpha \mathrm{Syn}^{\mathrm{A} 53 \mathrm{~T}}$. In a first step, potential changes of rapidly inactivating $\mathrm{K}^{+}$currents $\left(I_{\mathrm{A}}\right)$ were investigated (Fig. 2a). Quantification of $I_{\mathrm{A}}$ peak current densities revealed no significant changes in either $\alpha \mathrm{Syn}^{\mathrm{WT}}$ or in $\alpha \mathrm{Syn}^{\mathrm{A}}{ }^{53 \mathrm{~T}}$ overexpressing LC neurons compared to luc controls at any investigated time-point (Fig. 2b). Closer consideration of the current-voltage (I/V) relationships of $I_{\mathrm{A}}$ currents after 1, 3, 6 and 9 weeks post-injection supported the finding that these currents remained almost unchanged (Supplementary Fig. S2). Next, we analyzed peak current densities of delayed-rectifier/sustained $\mathrm{K}^{+}$ currents $\left(I_{\text {sus }}\right)$. However, peak current densities (Fig. 2c) as well as I/V relationships (Supplementary Fig. S2) remained unchanged. These results indicate that voltage-dependent $\mathrm{K}^{+}$channels of LC neurons are not influenced by aSyn overexpression and most likely do not contribute to the observed increase of excitability.

$\mathrm{Next}$, we investigated whether $\mathrm{Ca}^{2+}$ inward currents may be altered due to aSyn overexpression. As depicted in the average currents (Fig. 2d) and the I/V curves (Fig. 2e), at 9 weeks post-injection LC neurons of $\alpha \mathrm{Syn}^{\mathrm{WT}}$ and 
a
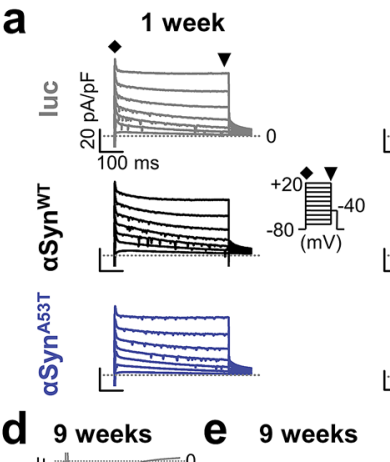
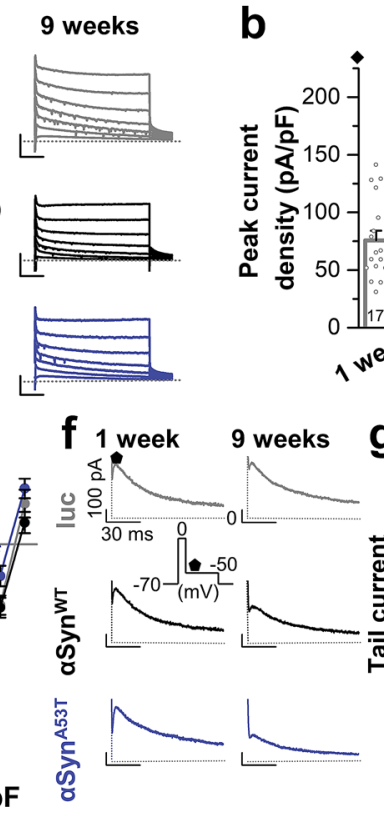
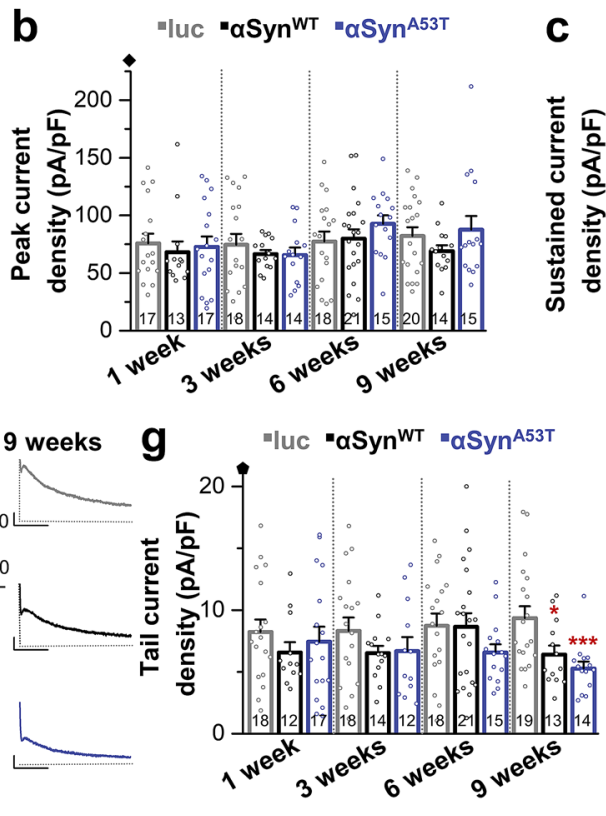
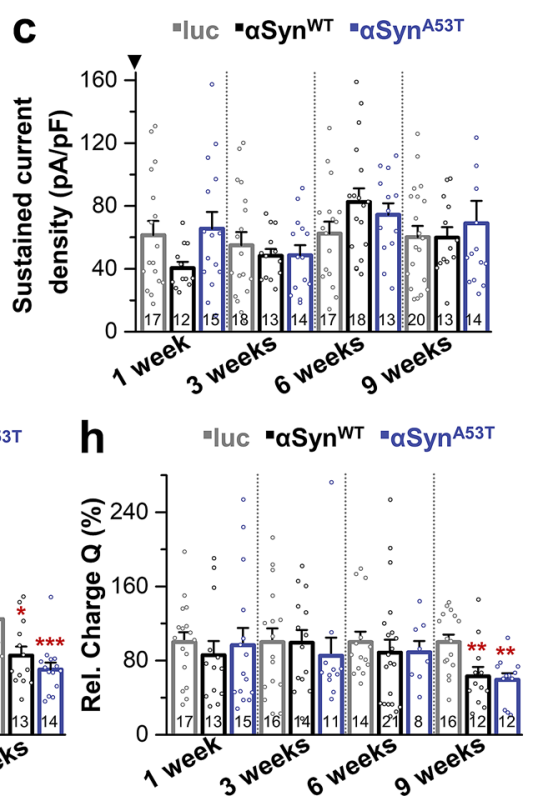

Figure 2. rAAV mediated $\alpha \mathrm{Syn}^{\mathrm{A} 53 \mathrm{~T}}$ overexpression induces alterations of $\mathrm{Ca}^{2+}$ - and small conductance $\mathrm{Ca}^{2+}$ activated $\mathrm{K}^{+}$currents in LC neurons. (a) Averaged whole-cell voltage clamp recordings of $\mathrm{K}^{+}$currents 1 and 9 weeks post-injection activated by a voltage protocol with $500 \mathrm{~ms}$ steps ranging from -70 to $+20 \mathrm{mV}$ starting from a holding potential of $-80 \mathrm{mV}$. (b) Peak current densities of $I_{\mathrm{A}}$ were ascertained from the start of each voltage step (indicated by rhombus in a) and quantified for each time-point after injection of viral vectors. (c) Peak current densities of $I_{\text {sus }}$ were ascertained from the end of each voltage step (indicated by triangle in a). For $I_{\text {sus }}$ current recordings the membrane potential was depolarized to $-40 \mathrm{mV}$ for $100 \mathrm{~ms}$ prior to voltage steps to remove $I_{\mathrm{A}}$ current components. (d) Average of $\mathrm{Ca}^{2+}$ current recordings 9 weeks after injection of viral vectors derived from a voltage step to $-10 \mathrm{mV}$. (e) I/V relationships of peak $\mathrm{Ca}^{2+}$ currents 9 weeks after injection, using the same voltage protocol as in $(\mathbf{a}) . \mathrm{Ca}^{2+}$ currents were significantly reduced 9 weeks post-injection in both $\alpha \mathrm{Syn}^{\mathrm{WT}}$ and $\alpha \mathrm{Syn}^{\mathrm{A}}{ }^{\mathrm{S} 3 \mathrm{~T}}$ injected mice (luc: $\mathrm{n}=14, \alpha \mathrm{Syn}^{\mathrm{WT}}: \mathrm{n}=28, \alpha \mathrm{Syn}^{\mathrm{A} 53 \mathrm{~T}}: \mathrm{n}=12$ ). (f) Representative $I_{\mathrm{AHP}}$ currents, activated by a two-pulse voltage clamp protocol as described previously ${ }^{22}$. The representative $I_{\mathrm{AHP}}$ currents 9 weeks after injection already display a drastic reduction in aSyn overexpressing neurons compared to luc. (g,h) Quantification of $I_{\mathrm{AHP}}$ current densities revealed significant reduction of AHP outward currents $(\mathbf{g})$ and relative charge $\mathrm{Q}(\mathbf{h})$ for $\alpha \mathrm{Syn}^{\mathrm{WT}}$ and $\alpha \mathrm{Syn}^{\mathrm{A} 53 \mathrm{~T}} 9$ weeks after injection. Data are presented as mean $\pm \mathrm{SEM}$ and also as individual data points. ${ }^{*} p<0.05 ;{ }^{* *} p<0.01$; ${ }^{* *} p<0.001$. [(b) 1 week: $\alpha$ Syn ${ }^{\mathrm{WT}}$, Mann-Whitney-U test vs. luc/aSyn ${ }^{\mathrm{AS3T}}$, unpaired Student's $t$-test vs. luc; 3 weeks: Welch's $t$-test vs. luc; 6 weeks: unpaired Student's $t$-test vs. luc; 9 weeks: Mann-Whitney-U test vs. luc. (c) 1 week and 6 weeks: Mann-Whitney-U test vs. corresponding luc; 3 weeks: $\alpha \mathrm{Syn}^{\mathrm{WT}}{ }^{\mathrm{T}}$, Welch's $t$-test vs. luc/ $\alpha$ Syn $^{\mathrm{ASBT}}$, Mann-Whitney-U test vs. luc; 9 weeks: $\alpha \mathrm{SSyn}^{\mathrm{WT}}$, unpaired Student's $t$-test vs. luc/aSyn ${ }^{\text {A53T }}$, Mann-Whitney-U test vs. luc. (e) Mann-Whitney-U test vs. luc. (g) 1 week: $\alpha$ Syn $^{\text {WT }}$, Mann-Whitney-U test vs. luc/aSyn ${ }^{\text {A53T }}$, unpaired Student's $t$-test vs. luc; 3 weeks: $\alpha$ Syn $^{\mathrm{WT}}$, Welch's $t$-test vs. luc/aSyn ${ }^{\mathrm{A} 53 \mathrm{~T}}$, unpaired Student's $t$-test vs. luc; 6 weeks: $\alpha \mathrm{Syn}^{\mathrm{WT}}$, unpaired Student's $t$-test vs. luc/ $/ \mathrm{SSyn}^{\mathrm{A} 53 \mathrm{~T}}$, Welch's $t$-test vs. luc; 9 weeks: $\alpha S_{y n}{ }^{\text {WT }}$, unpaired Student's $t$-test vs. luc/ $\alpha$ Syn $^{\mathrm{A} 53 \mathrm{~T}}$, Mann-Whitney-U test vs. luc. (h) 1 week and 6 weeks: Mann-Whitney-U test vs. corresponding luc; 3 weeks: $\alpha S y n{ }^{\mathrm{WT}}$, unpaired Student's $t$-test vs. luc/aSyn ${ }^{\mathrm{A} 53 \mathrm{~T}}$, Mann-Whitney-U test vs. luc; 9 weeks: unpaired Student's $t$-test vs. luc].

$\alpha$ Syn ${ }^{\mathrm{A53T}}$ overexpressing mice exhibited $\mathrm{Ca}^{2+}$ currents which were significantly reduced in a voltage range between $-20 \mathrm{mV}$ and $+10 \mathrm{mV}$ compared to luc controls (Fig. 2e). Subsequently, we investigated whether $\mathrm{Ca}^{2+}$ activated $\mathrm{K}^{+}$currents are altered due to aSyn overexpression and isolated currents flowing during the AHP of LC neurons $\left(I_{\mathrm{AHP}}\right)$, as it has already been shown that AHP currents are mainly mediated by SK channels ${ }^{22}$. The representative AHP currents in Fig. $2 \mathrm{f}$ display a drastic reduction of AHP current amplitudes after 9 weeks of overexpression in both aSyn groups compared to luc overexpressing neurons. Quantification of the $I_{\mathrm{AHP}}$ peak current densities confirmed a significant reduction after 9 weeks of both aSyn groups compared to luc overexpressing neurons (Fig. $2 \mathrm{~g}$ and Supplementary Fig. S3). Finally, as shown in Fig. $2 \mathrm{~h}$, we analyzed the percentage of the charge (Q), assessed from the integral of the AHP current recordings $\left(I_{\mathrm{AHP}}\right)$, at all time-points. The relative charge transfer through the AHP mediating channels was after 9 weeks of overexpression significantly decreased in both aSyn ${ }^{\mathrm{WT}}$ and $\alpha \mathrm{Syn}^{\mathrm{A}}{ }^{\mathrm{53T}}$ overexpressing LC neurons. Consistent with the more drastic increase of firing rates in $\alpha \mathrm{Syn}^{\mathrm{A}}{ }^{\mathrm{5} 3 \mathrm{~T}}$ injected mice, the effects on $\mathrm{Ca}^{2+}$ and AHP currents were also more pronounced in this group. Based on our previous observation that SK channels regulate LC neuron pacemaking by shaping of the AHP ${ }^{22}$, it is reasonable to assume that an overtime augmenting dysfunction of these channels, in combination with altered $\mathrm{Ca}^{2+}$ inward currents, may be causative for the increased excitability of $\alpha \mathrm{Syn}^{\mathrm{WT}}$ and $\alpha \mathrm{Syn}^{\mathrm{A} 33 \mathrm{~T}}$ overexpressing LC neurons. 

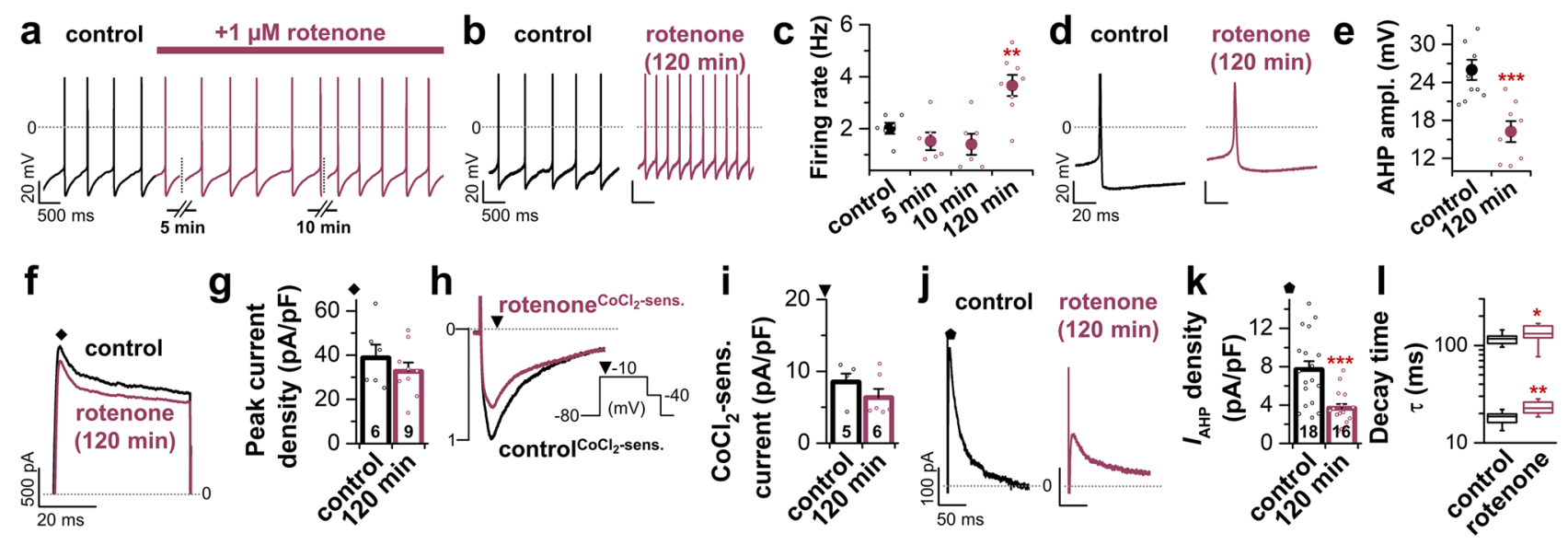

Figure 3. Electrophysiological alterations of LC neurons in an in vitro model of rotenone toxicity resemble those of aSyn overexpression. (a) Representative whole-cell spike train recording of a spontaneously active LC neuron in an acute brainstem slice derived from a C57BL/6 WT mouse. Wash-in of $1 \mu \mathrm{M}$ rotenone after $2 \mathrm{~min}$ of stable firing tendentially, but not significantly, reduced the discharge rate of LC neurons within $10 \mathrm{~min}$. (b) Example spike train recordings after slices were incubated for $2 \mathrm{~h}$ in standard ACSF (control, black) or ACSF $+1 \mu \mathrm{M}$ rotenone (red) prior to recording. (c) Quantification of spontaneous firing $5 \mathrm{~min}(\mathrm{n}=6), 10 \mathrm{~min}$ $(\mathrm{n}=6)$ and $2 \mathrm{~h}(\mathrm{n}=8)$ after exposure to $1 \mu \mathrm{M}$ rotenone. Pre-incubation of slices in rotenone led to a significant acceleration of the spontaneous firing rate compared to control $(n=6)$. (d) Representative AP traces of a control LC neuron (black) and an LC neuron pre-incubated in rotenone (red). (e) Quantification revealed a significant reduction of the AHP amplitude of LC neurons in slices incubated with rotenone compared to slices incubated in control ACSF (control: $n=10$, rotenone: $n=8$ ). (f) Representative whole-cell voltage clamp recordings of $I_{\mathrm{A}}$ currents activated by a $500 \mathrm{~ms}$ voltage step to $0 \mathrm{mV}$ from a holding potential of $-80 \mathrm{mV}$ (as depicted by rhombus in 2a). (g) Peak current densities of $\mathrm{K}^{+}$outward currents were ascertained from the start of the voltage step (indicated by rhombus in $\mathbf{f}$ ) and did not show a significant difference between control LC neurons and rotenone-exposed LC neurons. (h) $\mathrm{CoCl}_{2}$-sensitive $\mathrm{Ca}^{2+}$ inward currents derived from a voltage step to $-10 \mathrm{mV}$ from a holding potential of $-80 \mathrm{mV}$ as depicted in the inset. Either under control conditions (black) or after $2 \mathrm{~h}$ incubation in $1 \mu \mathrm{M}$ rotenone (red), $1 \mathrm{mM} \mathrm{CoCl}_{2}$ were washed in to block Cav channels. (i) Quantification of $\mathrm{CoCl}_{2}$-sensitive current densities revealed no significant difference between control and rotenone-exposed LC neurons. (j) Representative $I_{\mathrm{AHP}}$ currents, activated by the two-pulse voltage clamp protocol. (k) Quantification of $I_{\mathrm{AHP}}$ current densities revealed a drastic reduction of AHP outward currents after pre-incubation in rotenone. (l) In accordance with reduced medium $I_{\mathrm{AHP}}$ currents, the kinetic analysis of the decay time $(\tau)$ showed a slowing of the fast and medium decay time constants after $2 \mathrm{~h}$ rotenone treatment (control: $\mathrm{n}=10$, rotenone: $\mathrm{n}=11$ ). Box plots display median and $25 / 75$ percentile, whiskers indicate outliers. Rest of the data are presented as mean \pm SEM and also as individual data points. ${ }^{*} p<0.05 ;{ }^{* *} p<0.01 ;{ }^{* *} p<0.001$. [(c,e,g,l) unpaired Student's $t$-test vs. control. (i) Mann-Whitney-U test vs. control. (k) Welch's $t$-test vs. control].

Electrophysiological alterations of LC neurons in an in vitro model of rotenone toxicity resemble those of $\alpha$ Syn overexpression. In a next set of experiments, we aimed to investigate if these electrophysiological alterations are specific for the aSyn overexpression model, or whether they might be conserved in other PD models. To do so, we used an in vitro model with the neurotoxin rotenone. Rotenone, a commonly used pesticide ${ }^{26}$, inhibits complex I of the electron transport chain of the mitochondria, causing dopaminergic and noradrenergic neurodegeneration alongside with parkinsonian symptoms ${ }^{27-29}$. However, it is still unclear if and how rotenone influences the electrophysiological properties of LC neurons. We addressed this question using two different approaches: (1) by adding $1 \mu \mathrm{M}$ rotenone to the bath solution to assess direct effects, and (2) by incubating brainstem slices in $1 \mu \mathrm{M}$ rotenone for $120 \mathrm{~min}$ prior to electrophysiological recording.

In a first set of experiments, we performed spike train recordings in the whole-cell configuration to investigate acute effects of rotenone on the autonomous firing of LC neurons. While acute rotenone treatment for up to $10 \mathrm{~min}$ had no significant effect on LC firing rate (Fig. 3a,c), $120 \mathrm{~min}$ of preincubation with $1 \mu \mathrm{M}$ rotenone resulted in a significant acceleration of autonomous LC firing compared to the control condition (Fig. 3b,c). Furthermore, elevated LC pacemaking was associated with alterations of AP shape (Fig. 3d) and a significant reduction of the AHP amplitude (Fig. 3e). To elucidate the underlying mechanisms, we assessed the impact of rotenone incubation on different $\mathrm{K}^{+}$and $\mathrm{Ca}^{2+}$ currents. Peak current densities mediated by A-type $\mathrm{K}^{+}$channels (Fig. 3f) were not significantly different between control neurons and rotenone-exposed LC neurons (Fig. 3g). To check whether Cav channels were affected by rotenone, we blocked Cav channels with $1 \mathrm{mM} \mathrm{CoCl}_{2}$ and compared the $\mathrm{CoCl}_{2}$-sensitive current of rotenone-exposed neurons to that of controls (Fig. $3 \mathrm{~h}$ ). Even though peak current densities appeared reduced after rotenone application (Fig. 3h), quantification of $\mathrm{CoCl}_{2}$-sensitive current densities revealed no significant difference between control and rotenone-exposed LC neurons (Fig. 3i). Strikingly, similar as for the aSyn model, we observed a significant decrease of AHP currents (Fig. 3j,k), going alongside with altered $I_{\mathrm{AHP}}$ decay time constants $(\tau)$ (Fig. 3l). 


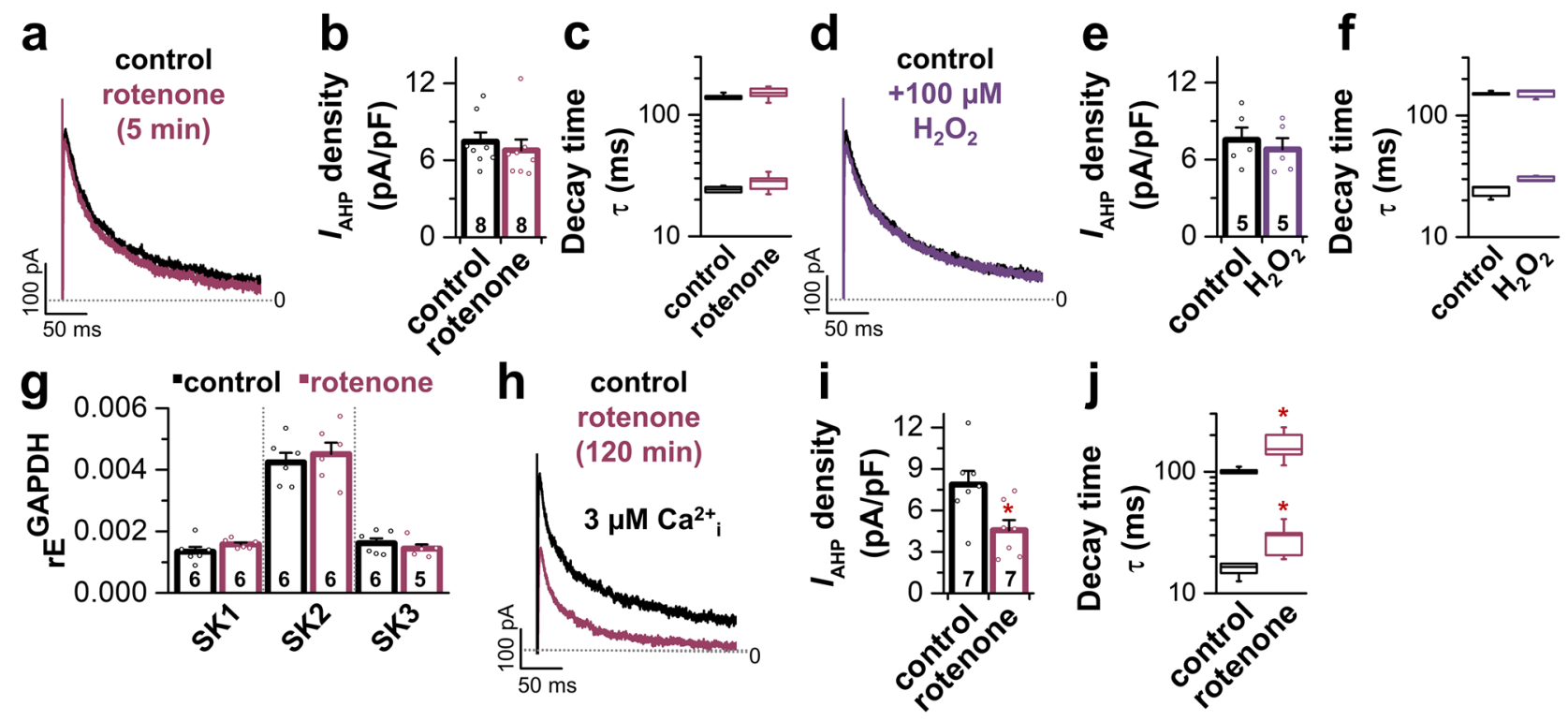

Figure 4. Rotenone-induced $I_{\mathrm{AHP}}$ alterations are independent of a direct rotenone effect, oxidative modulation, altered transcription and $\mathrm{Ca}^{2+}$ sensitivity. (a) Representative $I_{\mathrm{AHP}}$ recording of a LC neuron before (black) and 5 min after $1 \mu \mathrm{M}$ rotenone was washed in (red). (b,c) Acute rotenone exposure did not alter $I_{\mathrm{AHP}}$ current density $(\mathrm{n}=8)(\mathbf{b})$ or decay time $(\mathrm{n}=6)(\mathbf{c})$. (d) Representative $I_{\mathrm{AHP}}$ recording of a LC neuron before (black) and $5 \mathrm{~min}$ after $100 \mu \mathrm{M} \mathrm{H}_{2} \mathrm{O}_{2}$ was washed in (purple). (e,f) Wash-in of $100 \mu \mathrm{M} \mathrm{H}_{2} \mathrm{O}_{2}$ did not alter $I_{\mathrm{AHP}}$ peak current density (e) or current kinetic (f) within 5 min of wash-in $(n=5)$. (g) Relative expression (rE) levels of SK1, SK2 and SK3 channel subunits normalized to GAPDH. For expression analysis, LC neurons were collected out of acute brainstem slices ( $2 \mathrm{~h}$ incubation in ACSF (control) or $2 \mathrm{~h}$ incubation in $1 \mu \mathrm{M}$ rotenone). (h) Representative AHP currents in LC neurons of control slices and slices after $2 \mathrm{~h}$ pre-incubation with $1 \mu \mathrm{M}$ rotenone using high concentrations $(3 \mu \mathrm{M})$ of free $\mathrm{Ca}^{2+}$ in the pipette solution to achieve a full SK channel activation. (i,j) Use of high $\left[\mathrm{Ca}^{2+}\right]_{\mathrm{i}}$ did not restore the rotenone-induced reduction of $I_{\mathrm{AHP}}$ current density $(\mathrm{n}=7)(\mathbf{i})$ or current kinetic $(n=5)(j)$. Box plots display median and 25/75 percentile, whiskers indicate outliers. Rest of the data are represented as mean \pm SEM and also as individual data points. ${ }^{\star} p<0.05$. $[(\mathbf{b}, \mathbf{c})$ Mann-Whitney-U test vs. control. (e,f,g,i): unpaired Student's $t$-test vs. control. (j): Welch's $t$-test vs. control].

In summary, despite the fundamental differences between the aSyn and rotenone model, the electrophysiological changes induced by these two models are highly similar.

Reduced number of SK channels available at the membrane as a cause for the rotenone-induced $I_{\text {AHP }}$ reduction. In a next set of experiments, we aimed at shedding light on the mechanism of how longterm exposure to rotenone affects AHP/SK currents. First, to check whether rotenone acts as a direct modulator of SK channels, we recorded AHP currents of LC neurons before and $5 \mathrm{~min}$ after wash-in of $1 \mu \mathrm{M}$ rotenone. The quantification of peak current densities showed that acute rotenone exposure did not alter the $I_{\mathrm{AHP}}$ current density (Fig. 4a,b). Also, the decay of $I_{\mathrm{AHP}}$, which followed a bi-exponential time course, representing a "fast" and a "medium" component, was not changed due to the rotenone application (Fig. 4c). Rotenone is known to depolarize the mitochondrial membrane potential and to induce the release of $\operatorname{ROS}\left(\mathrm{H}_{2} \mathrm{O}_{2}\right)^{30}$. A study using adrenal medullary chromaffin cells demonstrated that SK2 and SK3 channels are modulated by the associated cellular redox state ${ }^{31}$. Given that murine LC neurons robustly express both SK2 and SK3 channels ${ }^{22}$, we subsequently investigated whether rotenone exerts an indirect effect on these channels by changing the cellular redox state. However, wash-in of $100 \mu \mathrm{M} \mathrm{H}_{2} \mathrm{O}_{2}$ did not alter the $I_{\mathrm{AHP}}$ peak current density or $\tau$ within 5 min of wash-in (Fig. 4d-f). Next, we elucidated whether long-term rotenone incubation of slices changed the expression level of the SK channel subunits SK1, SK2 and SK3. Hence, we performed qPCR analysis with RNA that was obtained from LC neurons, which were pipette-picked from slices that were incubated for 120 min either in ACSF (control) or in $1 \mu \mathrm{M}$ rotenone. The relative expression levels of SK1, SK2, and SK3 remained unchanged after $120 \mathrm{~min}$ rotenone exposure (Fig. $4 \mathrm{~g})$. As SK channels are regulated by changes of the intracellular $\mathrm{Ca}^{2+}\left(\left[\mathrm{Ca}^{2+}\right]_{\mathrm{i}}\right)$ concentration, we tested whether the rotenone-induced $I_{\mathrm{AHP}}$ reduction and changes in the decay time constants can be revoked by a high concentration of free $\mathrm{Ca}^{2+}(3 \mu \mathrm{M})$ in the pipette solution, a concentration high enough to fully activate all SK channels available at the plasma membrane. However, the use of high $\left[\mathrm{Ca}^{2+}\right]_{\mathrm{i}}$ did neither restore the rotenone-induced reduction of $I_{\mathrm{AHP}}$ current density (Fig. 4h,i versus Fig. 3j,k) nor the decelerated $\tau$ (Fig. $4 \mathrm{j}$ versus Fig. 31). Thus, as high $\left[\mathrm{Ca}^{2+}\right]_{\mathrm{i}}$ could not rescue the reduced $I_{\mathrm{AHP}}$ current amplitudes, we postulate that long-term rotenone treatment does not alter the gating or $\mathrm{Ca}^{2+}$-sensitivity of the channels and thus rotenone must act by reducing the quantity of SK channels on the plasma membrane of LC neurons. A reduced number of SK channels at the plasma membrane could explain the reduction of the $I_{\mathrm{AHP}}$ peak current density, changes to the kinetics of the $I_{\mathrm{AHP}}$ and the reduced AHP amplitude of action potentials of LC neurons after long-term rotenone treatment. 
SK channel activation with NS309 ameliorates rotenone-induced alterations of LC neurons. In a recent study, we have shown that pharmacological activation of SK channels by NS309 ${ }^{32}$ decreases LC pacemaking 22 . In addition, activation of SK channels with NS309 is associated with a reduction of the loss of dopaminergic neurons after rotenone $\mathrm{e}^{33,34}$ or glutamate treatment ${ }^{35,36}$. Therefore, we hypothesized that activation of SK channels by NS309 treatment would counteract the increased pacemaking caused by rotenone, by restoring macroscopic SK channel conductivity of LC neurons.

In a first set of experiments, we performed patch clamp recordings of LC neurons from slices that were either incubated for $2 \mathrm{~h}$ in ACSF (control), $1 \mu \mathrm{M}$ rotenone or $1 \mu \mathrm{M}$ rotenone $+20 \mu \mathrm{M}$ NS309 (Fig. 5a). Consistent with the results described above, we observed a two-fold increase of the firing rate due to the incubation with rotenone. Notably, simultaneous activation of SK channels with NS309 prevented the rotenone-induced acceleration of firing (Fig. 5b) and significantly diminished the rotenone-induced reduction of the AHP (Fig. 5c,d). In addition, analysis of $I_{\mathrm{AHP}}$ peak current densities showed that the marked rotenone-induced reduction of $I_{\mathrm{AHP}}$ was prevented by simultaneous administration of NS309 (Fig. 5e,f). In accordance with these results, preincubation with NS309 had a preventive effect on the decay time constants of $I_{\mathrm{AHP}}$ and the relative $I_{\mathrm{AHP}}$ charge, which resulted in values comparable to control (Fig. 5g,i). Quantification of the amplitude ratio of the decay time constants revealed a reduced "fast" component of $I_{\mathrm{AHP}}$ due to rotenone incubation, which was also restored to control values by coincubation with NS309 (Fig. 5h).

Next, we used primary LC neuronal cultures to investigate whether acute rotenone exposure alters the $\left[\mathrm{Ca}^{2+}\right]_{\mathrm{i}}$ level of LC neurons. It is known that the neurotoxic effect of rotenone is partly due to a dysregulated $\mathrm{Ca}^{2+} \mathrm{homeo}^{-}$ stasis and resulting oxidative stress ${ }^{37}$. Therefore, calcium imaging experiments in primary LC neurons were performed using the $\mathrm{Ca}^{2+}$ marker Fura-2 AM (Fig. 5j-1). To determine the $\left[\mathrm{Ca}^{2+}\right]_{\mathrm{i}}$, neurons were excited with wavelengths of $340 \mathrm{~nm}$ (reflecting $\mathrm{Ca}^{2+}$-bound fura-2) and $380 \mathrm{~nm}$ (reflecting free fura-2) and the fluorescence intensities were recorded. The ratio of these intensities ( $\mathrm{F}$ ratio) served as a measure of the $\left[\mathrm{Ca}^{2+}\right]_{\mathrm{i}}$ concentration. The representative images and quantification of the $\mathrm{F}$ ratio show that $5 \mathrm{~min}$ after application of $1 \mu \mathrm{M}$ rotenone, a drastic increase of $\left[\mathrm{Ca}^{2+}\right]_{\mathrm{i}}$ was detected in control cells, whereas cells pre-treated with $20 \mu \mathrm{M}$ NS309 did not show any alteration of fluorescence intensities after rotenone application (Fig. 5k,1). Hence, SK channel activation by NS309 protected primary LC neurons from rotenone-induced $\mathrm{Ca}^{2+}$ overload. Note that we have also performed control experiments, which show that the observed $\mathrm{Ca}^{2+}$ increase was not mediated by enhanced influx via plasmalemmal $\mathrm{Ca}^{2+}$ channels (Supplementary Fig. S4).

Moreover, we further investigated whether the activation of SK channels is not only beneficial for preventing electrophysiological changes of LC neurons, but also providing a neuroprotective effect. Therefore, the impact of SK channel activation on the rotenone-induced LC cell loss was evaluated with unbiased stereological quantification of TH-immunoreactive (TH-ir) LC neurons. The TH-ir LC cells were identified with DAB staining and Nissl counterstaining (Fig. $5 \mathrm{~m}$ ). As expected a significant degeneration of TH-ir LC cells was measured after $5 \mathrm{~h}$ incubation with rotenone (Fig. 5m,n). Strikingly, NS309 significantly diminished the rotenone-induced neuronal cell loss in the LC when applied simultaneously (Fig. 5n). These data show that the reduced SK channel conductivity at the plasma membrane cause increased action potential firing patterns which are associated with the loss of neurons in the LC. SK channel activators might therefore be beneficial to protect LC neurons at the early stages of PD.

\section{Discussion}

Dysfunction and neurodegeneration of noradrenergic LC neurons is a common feature of PD and other neurodegenerative diseases. Despite the early involvement in the prodromal phase of PD, only little is known about the functional consequences LC neurons face during the course of PD. Within this study, we aimed to elucidate the electrophysiological characteristics of LC neurons when rendered parkinsonian by either focal overexpression of human $\alpha \mathrm{Syn}^{\mathrm{WT}}$ or mutated $\alpha \mathrm{Syn}^{\mathrm{A} 53 \mathrm{~T}}$, or by application of rotenone, a complex I inhibitor known to induce PD pathology in mice ${ }^{38}$. Interestingly, despite the different nature of these two PD models, we observed very similar electrophysiological alterations and channel dysfunctions in both models. Three main conclusions can be drawn from our results. First, although SK channels do not control LC neuron pacemaking alone, their functionality is essential for slow and regular LC firing. Second, overexpression of aSyn as well as rotenone treatment disturb SK channel function and lead to an elevated AP firing frequency of LC neurons, which is associated with severe alterations of the AHP amplitude. Third, SK channels might present pharmacological targets for neuroprotective therapies given the fact that the SK channel activator NS309 showed neuroprotective effects in the rotenone model by decreasing the spontaneous LC firing rate to a physiological level and preventing subsequent neuronal loss in the LC following in vitro rotenone exposure. However, we did not provide an in vivo proof that SK channel activators are ultimately helpful against the PD progression. Future independent in vivo studies, addressing whether SK activators like NS309 can prevent Lewy body-like pathology in LC neurons, would strengthen our current hypothesis.

Given the fact that LC neurons have features which are very similar to that of the highly vulnerable dopaminergic SN neurons, including long and highly branched axons, a large number of neurotransmitter release sites, slow continuous autonomous pacemaking, and notable dendritic $\mathrm{Ca}^{2+}$ oscillations ${ }^{3,20}$, one can imagine that LC neurons also have high bioenergetic requirements to sustain their electrophysiological and functional activity. Moreover, previous work has shown that steady LC pacemaking triggers $\mathrm{Ca}^{2+}$-induced mitochondrial oxidant stress, which was significantly elevated during enhanced LC firing under hypercapnia ${ }^{20}$. Our results show that aSyn overexpression and long-term rotenone exposure also lead to elevated LC pacemaking, likely triggering similar effects. Based on the early formation of Lewy pathology in LC neurons, starting during the prodromal stage of PD, aSyn-induced hyperexcitability likely increases the bioenergetic burden of LC cells and could thereby be a major driver of subsequent LC neurodegeneration. 

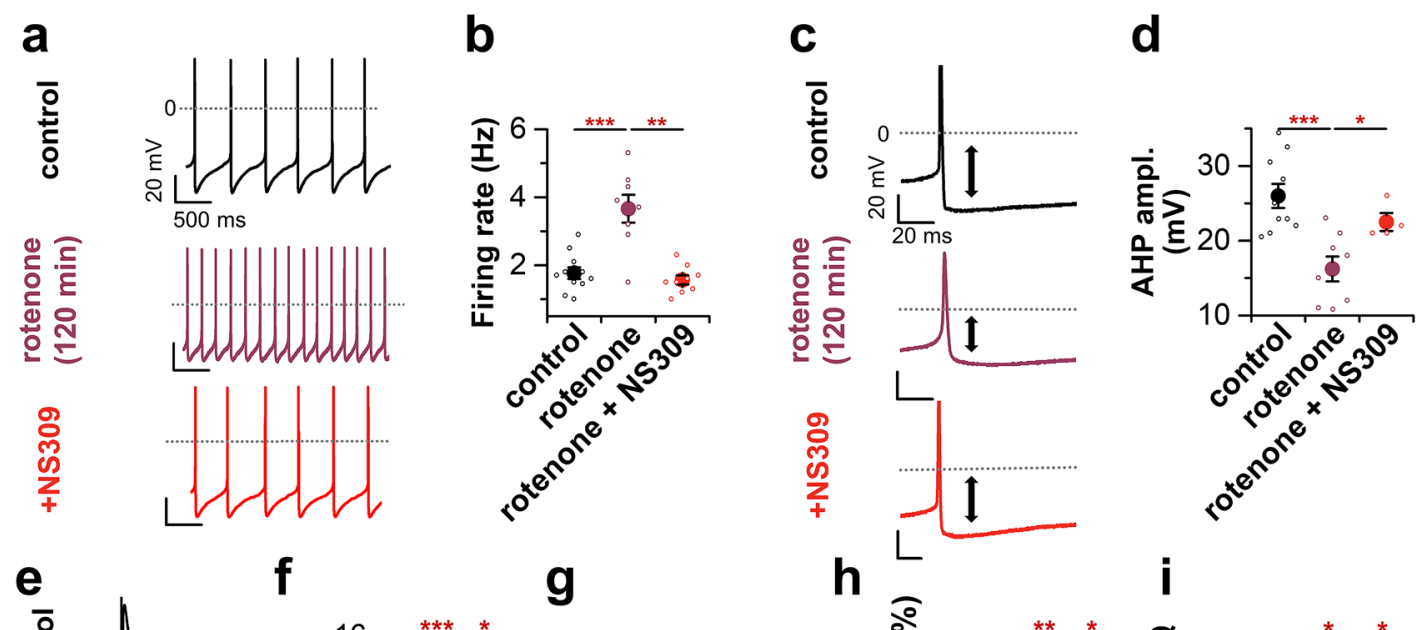

h
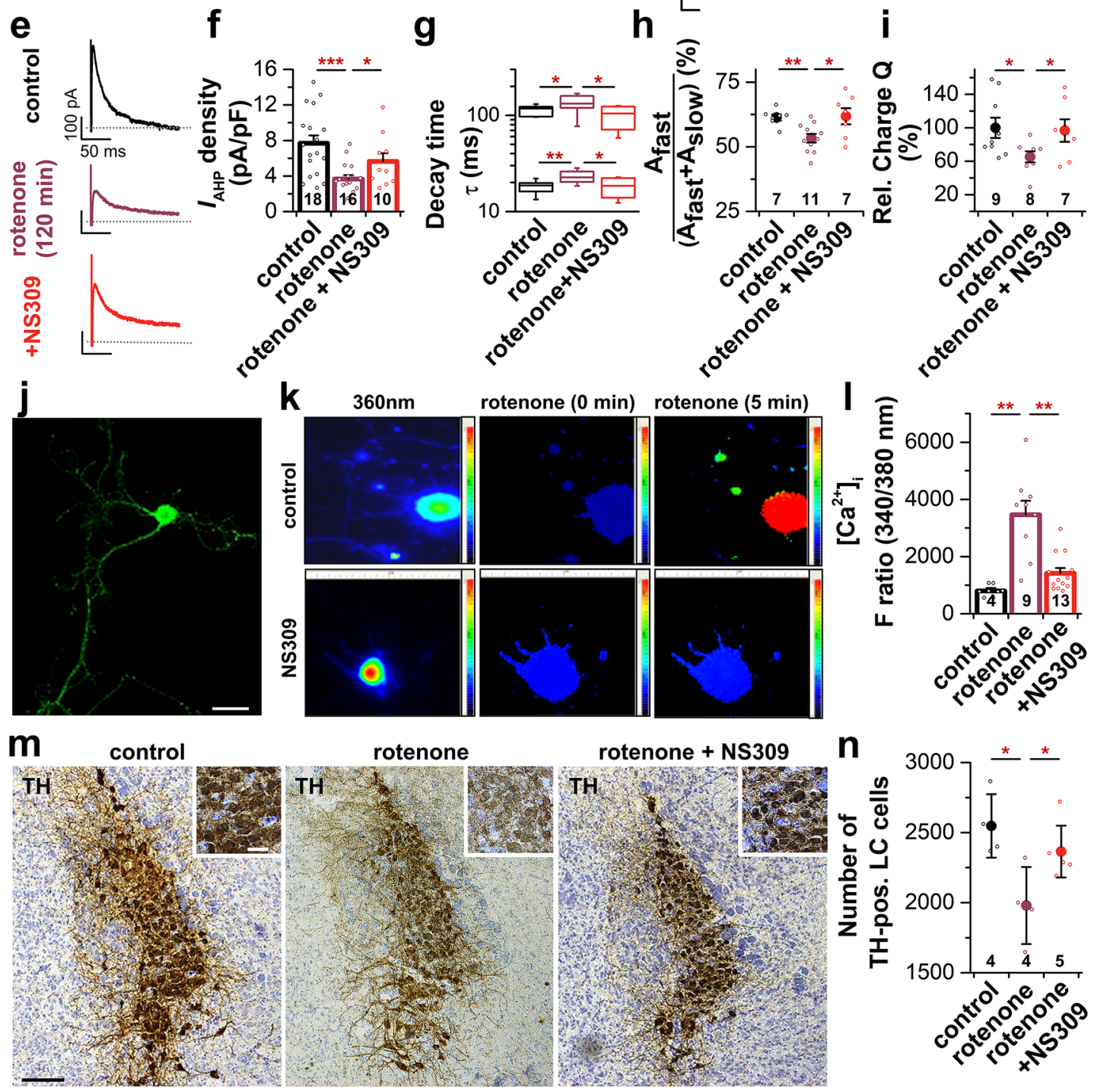
4Figure 5. SK channel activation with NS309 prevents the rotenone-induced increase of firing frequency, rotenone-induced $\mathrm{Ca}^{2+}$ overload in LC neurons and loss of TH-immunoreactive LC neurons. (a) Representative spike trains of LC neurons under control conditions (black) and after $2 \mathrm{~h}$ pre-incubation with $1 \mu \mathrm{M}$ rotenone (red) or $1 \mu \mathrm{M}$ rotenone and $20 \mu \mathrm{M}$ NS309 (bright red). (b) Quantification of AP firing frequency revealed that rotenone incubation $(n=8)$ led to a two-fold increase of the firing rate compared to control $(n=11)$, which was prevented by simultaneous activation of SK channels with NS309 (n=9). (c) Representative AP of an LC neuron after simultaneous incubation with rotenone and NS309. (d) Analysis of the AHP amplitude revealed that NS309 significantly diminished the rotenone-induced reduction of the AHP (control: $n=10$, rotenone: $\mathrm{n}=8$, rotenone + NS309: $\mathrm{n}=4$ ). (e) Representative voltage clamp recording of $I_{\mathrm{AHP}}$ currents after simultaneous incubation of the slice with rotenone and NS309. (f) The rotenone-induced reduction of $I_{\mathrm{AHP}}$ current density was prevented by simultaneous application of NS309. (g,h) $I_{\mathrm{AHP}}$ current kinetic analysis showed that the co-application of NS309 almost fully restored the rotenone-induced increase in decay time (control: $\mathrm{n}=10$, rotenone: $\mathrm{n}=10$, rotenone $+\mathrm{NS} 309: \mathrm{n}=7$ ). (i) Quantification of the net charge flux Q revealed that by simultaneous activation of SK channels with NS309 the rotenone-induced decrease in charge efflux was prevented. (j) Illustration of a primary LC neuron cultured for 1 week and stained with an anti-TH primary and Alexa488-conjugated secondary antibody. Scale bar: $100 \mu \mathrm{m}$. (k) Representative $\mathrm{Ca}^{2+}$ imaging of control cells (upper row) and cells pre-incubated with $20 \mu \mathrm{M}$ NS309 (lower row). $5 \mathrm{~min}$ after application of $1 \mu \mathrm{M}$ rotenone, a drastic increase of $\left[\mathrm{Ca}^{2+}\right]_{\mathrm{i}}$ (indicated by color change from blue to red) was detected in control cells, whereas NS309 pre-treated cells did not show any alteration of fluorescence intensities after rotenone application. (1) Quantification of intracellular $\mathrm{Ca}^{2+}$ by the ratio of fluorescence intensities of $\mathrm{Ca}^{2+}$-bound fura-2 (excitation at $340 \mathrm{~nm}$ wavelength) and free fura-2 (excitation at $380 \mathrm{~nm}$ wavelength) before application of rotenone (control), $5 \mathrm{~min}$ after application of rotenone and $5 \mathrm{~min}$ after application of rotenone following pre-incubation with $20 \mu \mathrm{M}$ NS309. SK channel activation with NS309 protected primary LC neurons from rotenone-induced $\mathrm{Ca}^{2+}$ overload. (m) Representative images of TH-immunoreactive (TH-ir) cells of the LC from the right hemisphere. Prior to sectioning, brainstem blocks containing the LC region were incubated in ACSF (control), ACSF $+1 \mu \mathrm{M}$ rotenone, or ACSF $+20 \mu \mathrm{M}$ NS309 $+1 \mu \mathrm{M}$ rotenone for 5 h. Scale bar: $100 \mu \mathrm{m}$, inset: $20 \mu \mathrm{m}$. (n) Unbiased stereology of TH-positive LC-neurons. SK channel activation with NS309 significantly reduced rotenone-induced neuronal loss in the LC. Box plots display median and 25/75 percentile, whiskers indicate outliers. Rest of the data are presented as mean \pm SEM and also as individual data points. ${ }^{*} p<0.05 ;{ }^{* *} p<0.01$; ${ }^{* * *} p<0.001$. [(b) rotenone vs. control, unpaired Student's $t$-test/rotenone vs. rotenone + NS309, Welch's $t$-test/ rotenone + NS309 vs. control, unpaired Student's $t$-test. (d,h) rotenone vs. control \& rotenone + NS309, unpaired Student's $t$-test/rotenone + NS309 vs. control, Welch's $t$-test. (f,i) rotenone vs. control, Welch's $t$-test/rotenone vs. rotenone + NS309, unpaired Student's $t$-test/rotenone + NS309 vs. control, unpaired Student's $t$-test. (g) decay

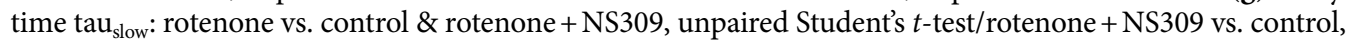
Welch's $t$-test; decay time tau fast $_{\text {: }}$ Student's $t$-test. (h) rotenone vs. control \& rotenone + NS309, unpaired Student's $t$-test/rotenone + NS309 vs. control, Welch's $t$-test. (l) rotenone vs. control, unpaired Student's $t$-test/rotenone vs. rotenone + NS309, Mann-Whitney-U test/rotenone + NS309 vs. control, Mann-Whitney-U test. (n) rotenone vs. control \& rotenone + NS309, unpaired Student's $t$-test].

During LC pacemaking, $\mathrm{Ca}^{2+}$ influx-likely via L- and T-type $\mathrm{Ca}^{2+}$ channels-activates SK channels which mediate $\mathrm{K}^{+}$outward currents that flow during the AHP $\left(I_{\mathrm{AHP}}\right)$ and thus SK channels, primarily SK2, control the medium AHP (mAHP) current ${ }^{22}$, the main current component that we recorded in our $I_{\mathrm{AHP}}$ mesurements. The $I_{\mathrm{AHP}}$ is critical for regulating autonomous pacemaking, as inhibition of SK channels leads to a decreased mAHP and increased neuronal excitability ${ }^{39}$. Our results are consistent with these previous observations and extend them in several aspects. aSyn overexpression, as well as long-term rotenone exposure, led to the same dysfunction of these channels: an increased AP frequency induced by a decreased post-hyperpolarization current $\left(I_{\mathrm{AHP}}\right)$ after long-term exposure. The most straightforward explanation of our results is that the reduced $I_{\mathrm{AHP}}$ results from a reduced or disturbed membrane trafficking of the SK channels. Although the precise mechanisms linking aSyn overexpression and rotenone exposure to SK channel dysfunction remain to be investigated in detail, support for this hypothesis comes from the fact that the transcripts levels of the SK channel subtypes SK1, SK2 and SK3 was not altered by rotenone. Most importantly, activation of the remaining SK channels with the SK channel activator NS309 $(20 \mu \mathrm{M})$ or high concentrations of intracellular $\mathrm{Ca}^{2+}(3 \mu \mathrm{M})$ were not able to restore the $I_{\mathrm{AHP}}$ current amplitudes, indicating that it is not an altered gating of the $\mathrm{Ca}^{2+}$ gated channels and that it must be a reduced number of $\mathrm{Ca}^{2+}$-dependent channels at the plasma. The medium $I_{\mathrm{AHP}}$ and the pacemaking of LC neurons critically depend on SK2 channels ${ }^{22}$. We found reduced $I_{\mathrm{AHP}}$ currents in LC neurons of two different PD models, which is in perfect agreement with studies reporting (1) that genetic deletion in the SK2 gene reduces $I_{\mathrm{AHP}}$, resulting in a PD mouse model ${ }^{40}$ and (2) that heterozygous loss-of-function mutations (haploinsufficiency) in KCNN2, encoding SK2, lead to novel autosomal dominant neurodevelopmental movement disorders ${ }^{41}$.

While the results of our rotenone exposure experiments showed a clear increase of firing frequency, mediated by SK channel dysfunction, Yee and colleagues ${ }^{42}$ observed a rotenone-induced, dose-dependent decrease of autonomous LC pacemaking. Importantly, in contrast to our long-term rotenone application over $120 \mathrm{~min}$, these experiments investigated acute effects of rotenone $(30 \mathrm{~min} \text { wash-in })^{37,42}$. The different time course could very well explain the differences between these two models. In fact, also in our experiments we observed a tendency for a firing frequency decrease after 5 and $10 \mathrm{~min}$ rotenone wash-in. We hypothesize that acute exposure of rotenone to LC neurons may result in intracellular $\mathrm{Ca}^{2+}$ overload, which could open $\mathrm{K}_{\mathrm{ATP}}$ channels and thereby decrease LC pacemaking. Another alternative explanation that needs to be considered is the bioenergetic effect of acute blockade of the electron transport chain (ETC) by rotenone-induced complex I inhibition. ETC inhibition leads to elevated levels of ADP which also trigger opening of $\mathrm{K}_{\mathrm{ATP}}$ channels and a decrease of firing frequency ${ }^{43}$. 
PD pathology within the noradrenergic LC system is tightly linked to several non-motor symptoms in the prodromal phase of PD patients ${ }^{44,45}$. However, it has been difficult to ascertain if these symptoms stem from early dysfunction of Lewy pathology harboring LC cells or from subsequent neurodegeneration and NE deficiency. Our observations, suggesting LC hyperactivity prior to neurodegeneration, seem to be of particular interest regarding at least two prominent and early non-motor symptoms, anxiety and depression. There is good evidence from previous studies which demonstrates that increased LC activity is anxiogenic ${ }^{46}$ and can cause depressive behavior in rodents ${ }^{4}$. Despite the need for further investigation, our results suggest that Lewy pathology formation in LC neurons could mediate anxiety and depression in prodromal PD patients. Given the clinical relevance of LC hyperactivity on the level of symptomatology and the probably negative pathophysiological consequences on the cellular side, SK channel activators may present new therapeutic targets to ameliorate LC hyperactivity in prodromal PD. The frequency and thus the oxidative stress in LC neurons could be arbitrarily titrated by SK2 activators and blockers independent of the pathogenesis (toxic or genetic) of the disease. Our data show that SK channel activation via NS309 protected primary LC neurons from rotenone-induced $\mathrm{Ca}^{2+}$ overload and significantly counteracted degeneration of LC cells in in situ brain tissue. However, one caveat that should be considered is the robust expression of SK channels in the dopaminergic SN. Since SK channel activators slow SN pacemaking and decrease dopamine release ${ }^{47}$, they could potentially worsen motor symptoms, especially in late stage PD patients. This highlights the need to further investigate if SK channel dysfunction is isolated to the noradrenergic LC, or a general feature of PD pathogenesis affecting also other vulnerable brain regions like the SN.

Due to the demographic change in the upcoming decades, the prevalence of PD will increase further if a disease modifying treatment is not discovered and implemented. Identification of new therapeutic targets, which would allow slowing, stopping or reversing the progression of PD already during the prodromal phase, presents an urgent need. By using a wide array of different electrophysiological readouts, we were able to link aSyn- and rotenone-induced LC pathology to dysfunction of SK channels, thereby providing a putative new target for therapeutic intervention in the prodromal phase of PD.

\section{Methods}

Animals. Wild-type, male C57BL/6 N mice were purchased from Charles River (Sulzfeld, Germany) and were 8 weeks old at the beginning of the experiments. Mice were housed in individually ventilated cages under a $12 \mathrm{~h} / 12 \mathrm{~h}$ light-dark cycle with ad libitum access to food and water. The health status of the animals was checked regularly by animal care technicians and a veterinarian. All procedures involving mice were conducted in accordance with protocols approved by the Regierungspräsidium Giessen, Germany V54-19 c 2015 h 01 MR 20/15 Nr. 66/2015. The investigation conforms to the principles outlined by the Declaration of Helsinki and to the guide for the Care and Use of Laboratory Animals (NIH Publication 85-23). Experiments adhered to guidelines from the Guide for the Care and Use of Laboratory Animals, U.S. Department of Health and Human Services.

Primary neuronal cultures from LC. Wild-type C57BL/6N pups (3-5 days old) were anesthetized with isoflurane, decapitated and brains were harvested in cold HBSS (Invitrogen). Coronal, $400 \mu \mathrm{m}$ thick brainstem sections containing the LC region were made using a Campden $7000 \mathrm{smz} 2$ vibratome (Campden Instruments). The microdissection followed a protocol described previously by Johnson et al. ${ }^{48}$. First, the LC region was removed from the brain with sterile razor blades. The microdissection was performed in Hibernate A medium (Invitrogen, Gibco) supplemented with $1 \times$ B27 (Invitrogen, Gibco) and $0.5 \mathrm{mM}$ glutamine (Invitrogen, Gibco). Subsequently, brain tissue was digested with trypsin (Invitrogen, Gibco) at $37^{\circ} \mathrm{C}$ for $30 \mathrm{~min}$, followed by the inactivation with trypsin inhibitor. Cells were dissociated and plated on coated dishes with polyethylenimine. The neuronal differentiation was performed in Neurobasal A medium (Invitrogen) supplemented with $1 \times$ B27, $0.5 \mathrm{mM}$ glutamine, $50 \mathrm{ng} / \mathrm{ml}$ BDNF (PeproTech) and $30 \mathrm{ng} / \mathrm{ml}$ GDNF (Sigma). Cultures were left in the incubator at $37{ }^{\circ} \mathrm{C}$ at $5 \% \mathrm{CO}_{2}$ for at least 7 days to mature with a media change twice a week (one quarter of the medium) with fresh Neurobasal A, $1 \times$ B27 and $0.5 \mathrm{mM}$ glutamine without BDNF and GDNF.

rAAV vectors and stereotactic injection. Recombinant adeno-associated viral (rAAV) vectors, serotype 1/2, were used to overexpress either human wild-type (WT)- $\alpha$ Syn (rAAV1/2-CMV/CBA-human-WTaSyn-WPRE-BGH-pA (rAAV1/2- SSyn $^{\mathrm{WT}}$ ); viral titer $5.1 \times 10^{12} \mathrm{gp} / \mathrm{ml}$, purchased from GeneDetect) or human mutant-A53T- $\alpha$ Syn (rAAV1/2-CMV/CBA-human-A53T- $\alpha$ Syn-WPRE-BGH-pA (rAAV1/2- $\alpha$ Syn $^{\text {A53T }}$ ); viral titer $5.1 \times 10^{12} \mathrm{gp} / \mathrm{ml}$, purchased from GeneDetect) and-as control-luciferase (rAAV1/2-CMV/CBA-luciferase-WPRE-BGH-pA (rAAV1/2-luc), viral titer $5.0 \times 10^{12} \mathrm{gp} / \mathrm{ml}$, purchased from GeneDetect). Expression of target proteins was driven by a chicken beta actin (CBA) promoter combined with a cytomegalovirus (CMV) immediate early enhancer sequence. To assess a high transcription rate, the woodchuck post-transcriptional regulatory element (WPRE) was included into the vectors ${ }^{49,50}$. For stereotactic injection of the rAAV vectors into the right LC region, mice were anesthetized with $100 \mathrm{mg} / \mathrm{kg}$ ketamine and $5 \mathrm{mg} / \mathrm{kg}$ xylazine. A volume of $1.25 \mu \mathrm{l}$ of each vector was injected with a velocity of $125 \mathrm{nl} / \mathrm{min}$ using a microinjector (UltraMicro Pump UMP3, World Precision Instruments) based on the following coordinates: medio-lateral $-0.9 \mathrm{~mm}$, antero-posterior $-5.4 \mathrm{~mm}$ and dorso-ventral $-3.65 \mathrm{~mm}$ relative to Bregma $^{51}$.

Electrophysiology. For slice preparation, mice were anesthetized with isoflurane, decapitated and brains were removed rapidly. Mice older than 30 days were injected intra-peritoneally with ketamine/xylazine (SigmaAldrich) anesthesia (260 and $40 \mathrm{mg}$ per kg body weight) and perfused transcardially with an ice-cold preparation solution composed of (in mM): $2.5 \mathrm{KCl}, 1.25 \mathrm{NaH}_{2} \mathrm{PO}_{4}, 10 \mathrm{MgSO}_{4}, 20$ PIPES, 10 glucose, 200 saccharose, and $0.5 \mathrm{CaCl}_{2}, \mathrm{pH} 7.35$ with $\mathrm{NaOH}$ prior to decapitation. Coronal sections (180-200 $\mu \mathrm{m}$ thick) were made using 
a Campden 7000smz-2 vibratome (Campden Instruments) in ice-cold preparation solution. After sectioning, slices were maintained in artificial cerebrospinal fluid (ACSF) composed of (in mM): $125 \mathrm{NaCl}, 2.5 \mathrm{KCl}, 25$ $\mathrm{NaHCO}_{3}, 1.25 \mathrm{NaH}_{2} \mathrm{PO}_{4}, 2 \mathrm{CaCl}_{2}, 1 \mathrm{MgCl}_{2}$ and 25 glucose, equilibrated to $\mathrm{pH} 7.4$ with $95 \% \mathrm{O}_{2} / 5 \% \mathrm{CO}_{2}$ at $30{ }^{\circ} \mathrm{C}$. Following a $30 \mathrm{~min}$ resting time, slices were kept at room temperature for up to $5 \mathrm{~h}$.

For patch clamp recordings, slices were transferred to a recording chamber mounted on a Zeiss Examiner.D1 microscope (Carl Zeiss Microscopy, LLC, United States) with a $40 \times / 0.75$ phase contrast, water immersion objective and a Zeiss AxioCam MRm camera (Carl Zeiss Microscopy, LLC, United States). During recordings, slices were continuously perfused with ACSF, unless specified otherwise. During recording, LC neurons in brainstem slices were identified based on their localization at the edge of the fourth ventricle and large somata. All recordings were performed at room temperature with patch pipettes prepared from borosilicate glass capillaries GB 150 TF- 8P (Science Products, Hofheim, Germany) with tip resistances between 3 and $5 \mathrm{M} \Omega$. Conventional tight seal whole-cell voltage clamp or current clamp experiments were conducted with ACSF as external solution and an internal solution containing (in mM): $135 \mathrm{~K}$-Gluconate, $5 \mathrm{KCl}, 10 \mathrm{HEPES}, 0.1$ EGTA, $2 \mathrm{MgCl}_{2}, 0.2 \mathrm{Li}_{2} \mathrm{GTP}, 2$ MgATP, 3 neurobiotin and adjusted to $\mathrm{pH} 7.35$ with $\mathrm{KOH}$. For measurements of $\mathrm{Ca}^{2+}$ currents a modified ACSF with a lower $\mathrm{CaCl}_{2}$ concentration of $1 \mathrm{mM}$, and an internal pipette solution containing (in $\mathrm{mM}$ ): $111 \mathrm{CsCH}_{3} \mathrm{SO}_{3}$, $12.5 \mathrm{CsCl}, 1 \mathrm{MgCl}_{2}, 1 \mathrm{CaCl}_{2}, 10 \mathrm{HEPES}, 1 \mathrm{EGTA}, 2 \mathrm{MgATP}, 0.21 \mathrm{Na}_{2} \mathrm{GTP}$, adjusted to $\mathrm{pH}$ of 7.35 with $\mathrm{CsOH}$ were used. Neurobiotin (NB, Vector Laboratories) was used to label recorded neurons. To isolate autonomous spiking, all recordings were performed in the presence of synaptic blockers ( $1 \mu \mathrm{M}$ CGP, $10 \mu \mathrm{M}$ gabazine, $10 \mu \mathrm{M}$ AP-5, $5 \mu \mathrm{M}$ NBQX).

All patch clamp recordings were made using an Axopatch 200B amplifier (Molecular Devices, Sunnyvale CA, USA) and Clampex 10.0 software (pClamp10, Molecular Devices, Sunnyvale CA, USA). Data were digitized at $10 \mathrm{kHz}$ with a Digidata 1440 A digitizer (Molecular Devices, Sunnyvale CA, USA), filtered at $1-5 \mathrm{kHz}$ and electrode capacitance was compensated. Membrane and tip resistances were checked after each measurement and recordings with significant changes of these values (i.e. membrane resistances $<1 \mathrm{G} \Omega$ and/or tip resistances of $>20$ $\mathrm{M} \Omega$ ) were excluded from further analyses. For application of drugs respective stock solutions were diluted in ACSF/ modified ASCF freshly on the day of recording. As a prerequisite for application of drugs, a stable recording of at least $2 \mathrm{~min}$ was defined. Experiments were repeated after at least $10 \mathrm{ml}$ of drug containing ACSF solution had run through the perfusion system $(\sim 2 \mathrm{~min})$. Data were analyzed with ClampFit10 (Molecular Devices, Sunnyvale CA, USA). Drugs were stored as DMSO stocks and final DMSO concentration did not exceed $0.1 \%$.

Immunofluorescence staining. For immunofluorescent co-staining of NB, alpha-synuclein ( $\alpha$ Syn) and tyrosine hydroxylase $(\mathrm{TH})$ at the end of each patch clamp recording slices were transferred to a fixation solution composed of $4 \%$ paraformaldehyde (PFA) in $0.1 \mathrm{M}$ phosphate buffer (PB). After fixation overnight, slices were stored in storing solution containing $30 \%$ sucrose in $0.1 \mathrm{M}$ PBS. Before staining $200 \mu \mathrm{m}$ thick slices were cut thinner $(40 \mu \mathrm{m})$ using a cryostat microtome (Leica CM3050 S, Nussloch, Germany). Sections were washed in $0.1 \mathrm{M}$ PB and blocked in $10 \%$ normal donkey serum with $0.3 \%$ Triton X-100 in $0.1 \mathrm{M}$ PB for $1 \mathrm{~h}$ followed by overnight incubation with primary antibodies for TH (1:1000, Merck Millipore, Cat\# AB152, RRID: AB_390204) and aSyn (1:1000, ThermoFisher Scientific, Cat\# AHB0261, RRID: AB_2536241) at $4{ }^{\circ} \mathrm{C}$. Subsequently, sections were washed in $0.1 \mathrm{M} \mathrm{PB}$ containing $0.3 \%$ Triton X-100 and then incubated with fluorophore-conjugated, species-specific secondary antibodies Cy3 conjugated donkey anti-mouse (1:1000, Jackson ImmunoResearch, Cat\# 715-165-150, RRID: AB_2340813), Alexa488 conjugated donkey anti-rabbit (1:1000, Invitrogen, Cat\# A-21206, RRID: AB_2535792) and Alexa 647-conjugated streptavidin (1:1000, Jackson ImmunoResearch, Cat\# 016-600084, RRID: AB_2341101) for $2 \mathrm{~h}$ at room temperature in $0.1 \mathrm{M}$ PB containing $0.3 \%$ Triton X-100 and $10 \%$ normal donkey serum. Before mounting, sections were washed for $25 \mathrm{~min}$ in $0.1 \mathrm{M} \mathrm{PB}$ containing $0.3 \%$ Triton $\mathrm{X}-100$. Images were acquired using an AxioImager M2 microscope (Carl Zeiss Microscopy, LLC, United States) equipped with an ORCA-Flash4.0 LT CMOS camera (Hamamatsu C11440-42U).

Real time qPCR. For quantitative (q-) PCR experiments, single LC neurons were pulled out of acute brainstem slices with a modified patch pipette as described previously ${ }^{21}$. RNA isolation was performed with the RN-easy Protect Mini Kit (Qiagen). Reverse transcription (RT) was performed with random hexamers (Roche) and Superscript II reverse transcriptase (Invitrogen) according to the instructions of the manufacturer. For further analyses, only cDNA pools that were negative for GAD, but positive for GAPDH and dopamine-betahydroxylase $(\mathrm{DBH})$ were used.

Intron-spanning primers were designed to generate PCR products of about 110 base pairs:

GAPDH for 5'-ACTTCAACAGCAACTCCCACTCT-3', GAPDH rev 5'-GCTGTAGCCGTATTCATTGTCATA-3';

DBH for 5'-ACTATGTGCACTACTACCCCCAGA-3',

DBH rev 5'-CTCCTCACTGCTGAACCTGTTTAC-3';

GAD for 5'-GGAGCGGATCCTAATACTACCAA-3',

GAD rev 5'-GTAAGAAGCCACAGATCTTCAGG-3';

SK1 for 5'-GAGAAACACGTGCACAACTTCAT-3',

SK1 rev 5'-CAGCTCTGACACCACCTCATATG-3';

SK2 for 5'-GAATGACCAAGCAAATACCCTAGT-3',

SK2 rev 5'-GTGACGATCCTTTTCTCAAAGTCT-3';

SK3 for 5' - GAAAAGAGAAAGCGACTGAGTGAC-3',

SK3 rev 5'-CATGGAATCCTTTGAGTACAAACC-3'; 
qPCRs were performed as described previously ${ }^{22}$. Briefly, the Platinum SYBR Green qPCR SuperMix-UDG (Invitrogen) was used and reaction mixtures were preheated at $50^{\circ} \mathrm{C}$ and at $95^{\circ} \mathrm{C}$ for $2 \mathrm{~min}$ each, followed by 40 cycles at $95^{\circ} \mathrm{C}$ for $15 \mathrm{~s}, 60^{\circ} \mathrm{C}$ for $30 \mathrm{~s}$, and $72{ }^{\circ} \mathrm{C}$ for $30 \mathrm{~s}$. Emitted fluorescence was detected online using a Mx3000P real-time PCR system (Stratagene). The amplification products for all primer pairs were confirmed by sequencing, no template control and dissociation curve analysis. Amplification efficiency was determined by analyzing the slope of a Ct/log (template concentration) plot. For normalization, primers for GAPDH were used $\left(\mathrm{rE}^{\mathrm{GAPDH}}=1 / 2^{\Delta \mathrm{Ct}}\right)$. $\mathrm{qPCR}$ reactions were performed in duplicate or triplicate, and control experiments in the absence of cDNA (n.c.) were included. Three independent qPCR experiments were performed.

Calcium imaging of primary LC neurons. Primary neuronal LC cells were incubated with $2 \mu$ M FURA-2 $\mathrm{AM}$ for $30 \mathrm{~min}$ at $37^{\circ} \mathrm{C}$ in HEPES-ringer buffer (HRB), containing (in $\mathrm{mM}$ ): $136.4 \mathrm{NaCl}, 5.6 \mathrm{KCl}, 1 \mathrm{MgCl}_{2}, 2.2$ $\mathrm{CaCl}_{2}, 10$ HEPES, 5 glucose and $0.1 \%$ BSA, pH 7.4. Images were acquired using a Polychrome II monochromator and an IMAGO CCD camera (Till Photonics, Martinsried, Germany) coupled to an inverted microscope (IX70; Olympus, Hamburg, Germany). Images were collected with a $20 \times 0.8$ numerical aperture (NA) oil immersion objective. Fluorescence intensities were monitored from single cells excited at the two wavelengths (F340 and F380) and the emission wavelength used was $510 \mathrm{~nm}$. An increase in intracellular $\mathrm{Ca}^{2+}$ was reflected by a fluorescence increase when exciting at $340 \mathrm{~nm}$ and a corresponding decrease when excited at $380 \mathrm{~nm}$. F340 and F380 were recorded separately and combined (fluorescence ratio: $r=F 340 / F 380$ ) after background subtraction (fluorescence of a cell-free area).

Stereology. To quantify TH-immunoreactive LC neurons, $30 \mu \mathrm{m}$ thick tissue sections were stained against TH with 3,3-diaminobenzidine (DAB), counterstained with cresyl-violet and the optical fractionator workflow (StereoInvestigator version 8, MicroBrightField Biosciences) was used ${ }^{18}$. For DAB stainings, free-floating brainstem sections containing the LC region were washed three times in $0.1 \mathrm{M} \mathrm{PB}$ and quenched with $3 \% \mathrm{H}_{2} \mathrm{O}_{2}$ and $10 \%$ methanol for $15 \mathrm{~min}$. Then, sections were blocked with $5 \%$ normal donkey serum $/ 0.3 \%$ Triton X-100 in $0.1 \mathrm{M}$ PB for $1 \mathrm{~h}$ followed by overnight incubation with a primary anti-TH antibody $(1: 1000$, Merck Millipore, Cat\# AB152, RRID: AB_390204) at $4{ }^{\circ} \mathrm{C}$ in the same blocking solution. Subsequently, sections were washed in $0.1 \mathrm{M}$ PB for $20 \mathrm{~min}$ and then incubated with a biotinylated anti-rabbit secondary antibody (1:1000, Jackson ImmunoResearch, Cat\# 711-065-152, RRID: AB_2340593) for $1 \mathrm{~h}$, followed by incubation in avidin-biotinperoxidase solution (ABC Elite, Vector Laboratories) for $1 \mathrm{~h}$ before initiating the color reaction with $5 \% \mathrm{DAB}$ (Serva), diluted in $0.1 \mathrm{M} \mathrm{PB}$ with $0.02 \% \mathrm{H}_{2} \mathrm{O}_{2}$. DAB-stained sections were counterstained with cresyl-violet. Brightfield images were acquired using an AxioImager M2 microscope (Carl Zeiss Microscopy, LLC, United States) equipped with an Axiocam 506 color camera (Carl Zeiss Microscopy, LLC, United States).

To quantify LC cell numbers four systematically, randomly acquired sections per animal containing the complete rostro-caudal extent of the LC region were analyzed. First, contours including all TH-positive neurons of the LC were drawn in $5 \times$ magnification using a Microphot-FX microscope (Nikon, Tokyo, Japan). Counting was performed in $40 \times$ magnification using the following parameters: grid size $100 \times 100 \mu \mathrm{m}$, counting frame $85 \times 85 \mu \mathrm{m}$, and guard zones $2 \mu \mathrm{m}$.

Data and statistical analysis. Data are reported as mean \pm standard error of the mean (SEM) and were analyzed using Excel (Microsoft, Washington, USA), Clampfit (Molecular Devices, California, USA) and OriginPro (OriginLab Corp., Guangzhou, China. Data sets were tested for normality using the Shapiro Wilk test and for variance homogeneity using the one-way ANOVA-Levene's test. In normally distributed data sets, statistical significance was calculated using paired or unpaired Student's $t$-test, or Welch's $t$-test depending on the variance. Otherwise, non-parametric statistical tests, e.g. the Mann-Whitney-U test or the Mood's median test were used depending on the variance. Decay time constants $(\tau)$ were determined by fitting the $I_{\mathrm{AHP}}$ current tails to a biexponential decay function. Box plots display median, $25^{\text {th }}$ and $75^{\text {th }}$ percentile, whiskers indicate the total range. All experiments were performed at room temperature $\left(21-24^{\circ} \mathrm{C}\right)$. ${ }^{*}$ indicates $p<0.05$; ${ }^{* *}$ when $p<0.01$ and ${ }^{* * *}$ when $p<0.001$. Only significant changes are indicated in the figures and a lack of asterisks next to the bar graphs shows that there are no significant changes.

Materials. All drugs or chemicals were obtained from Sigma-Aldrich, Carl Roth, Honeywell Fluka or biomol.

Ethical approval. All animal research was ethically approved by the governmental authority (Regierungspräsidium Giessen, Germany reference V54-19 c 2015 h 01 MR 20/15 Nr. 66/2015). All procedures involving mice were performed in accordance with relevant guidelines and regulations. The study is reported in accordance with ARRIVE guidelines.

\section{Data availability}

The data supporting the findings of this study are available from the corresponding author upon reasonable request.

Received: 8 November 2021; Accepted: 7 February 2022

Published online: 24 February 2022 


\section{References}

1. Braak, H. et al. Staging of brain pathology related to sporadic Parkinson's disease. Neurobiol. Aging 24, 197-211 (2003).

2. Surmeier, D. J., Obeso, J. A. \& Halliday, G. M. Selective neuronal vulnerability in Parkinson disease. Nat. Rev. Neurosci 18, $101-113$. https://doi.org/10.1038/nrn.2016.178 (2017).

3. Oertel, W. H., Henrich, M. T., Janzen, A. \& Geibl, F. F. The locus coeruleus: Another vulnerability target in Parkinson's disease. Mov. Disord. 34, 1423-1429. https://doi.org/10.1002/mds.27785 (2019).

4. Weinshenker, D. Long road to ruin: Noradrenergic dysfunction in neurodegenerative disease. Trends Neurosci. 41, 211-223. https:// doi.org/10.1016/j.tins.2018.01.010 (2018).

5. Knudsen, K. et al. In-vivo staging of pathology in REM sleep behaviour disorder: A multimodality imaging case-control study. Lancet Neurol. 17, 618-628. https://doi.org/10.1016/S1474-4422(18)30162-5 (2018).

6. Giguère, N., Burke Nanni, S. \& Trudeau, L. E. On cell loss and selective vulnerability of neuronal populations in Parkinson's disease. Front. Neurol. 9, 455. https://doi.org/10.3389/fneur.2018.00455 (2018).

7. Zarow, C., Lyness, S. A., Mortimer, J. A. \& Chui, H. C. Neuronal loss is greater in the locus coeruleus than nucleus basalis and substantia nigra in Alzheimer and Parkinson diseases. Arch. Neurol. 60, 337-341 (2003).

8. Bremner, J. D., Krystal, J. H., Southwick, S. M. \& Charney, D. S. Noradrenergic mechanisms in stress and anxiety: I. Preclinical studies. Synapse 23, 28-38. https://doi.org/10.1002/(sici)1098-2396(199605)23:1\%3c28::aid-syn4\%3e3.0.co;2-j (1996).

9. Remy, P., Doder, M., Lees, A., Turjanski, N. \& Brooks, D. Depression in Parkinson's disease: loss of dopamine and noradrenaline innervation in the limbic system. Brain 128, 1314-1322. https://doi.org/10.1093/brain/awh445 (2005).

10. Feinstein, D. L., Kalinin, S. \& Braun, D. Causes, consequences, and cures for neuroinflammation mediated via the locus coeruleus: Noradrenergic signaling system. J. Neurochem. 139(Suppl 2), 154-178. https://doi.org/10.1111/jnc.13447 (2016).

11. Qian, L. et al. $\beta_{2}$-adrenergic receptor activation prevents rodent dopaminergic neurotoxicity by inhibiting microglia via a novel signaling pathway. J. Immunol. 186, 4443-4454. https://doi.org/10.4049/jimmunol.1002449 (2011).

12. Sabogal-Guáqueta, A. M. et al. Microglia alterations in neurodegenerative diseases and their modeling with human induced pluripotent stem cell and other platforms. Prog. Neurobiol. 190, 101805. https://doi.org/10.1016/j.pneurobio.2020.101805 (2020).

13. Fornai, F. et al. Norepinephrine loss selectively enhances chronic nigrostriatal dopamine depletion in mice and rats. Brain Res. 735, 349-353 (1996)

14. Ferrucci, M. et al. Noradrenergic loss enhances MDMA toxicity and induces ubiquitin-positive striatal whorls. Neurol. Sci. 23(Suppl 2), S75-76. https://doi.org/10.1007/s100720200077 (2002).

15. Bing, G., Zhang, Y., Watanabe, Y., McEwen, B. S. \& Stone, E. A. Locus coeruleus lesions potentiate neurotoxic effects of MPTP in dopaminergic neurons of the substantia nigra. Brain Res. 668, 261-265 (1994).

16. Kilbourn, M. R., Sherman, P. \& Abbott, L. C. Reduced MPTP neurotoxicity in striatum of the mutant mouse tottering. Synapse 30, 205-210. https://doi.org/10.1002/(sici)1098-2396(199810)30:2\%3c205::aid-syn10\%3e3.0.co;2-0 (1998).

17. Kreiner, G. et al. Stimulation of noradrenergic transmission by reboxetine is beneficial for a mouse model of progressive parkinsonism. Sci. Rep. 9, 5262. https://doi.org/10.1038/s41598-019-41756-3 (2019).

18. Henrich, M. T. et al. A53T- $\alpha$-synuclein overexpression in murine locus coeruleus induces Parkinson's disease-like pathology in neurons and glia. Acta Neuropathol. Commun. 6, 39. https://doi.org/10.1186/s40478-018-0541-1 (2018).

19. Osmanović, S. S., Shefner, S. A. \& Brodie, M. S. Functional significance of the apamin-sensitive conductance in rat locus coeruleus neurons. Brain Res. 530, 283-289 (1990).

20. Sanchez-Padilla, J. et al. Mitochondrial oxidant stress in locus coeruleus is regulated by activity and nitric oxide synthase. Nat. Neurosci. 17, 832-840. https://doi.org/10.1038/nn.3717 (2014).

21. Matschke, L. A. et al. A concerted action of L- and T-type $\mathrm{Ca}^{2+}$ channels regulates locus coeruleus pacemaking. Mol. Cell. Neurosci. 68, 293-302. https://doi.org/10.1016/j.mcn.2015.08.012 (2015).

22. Matschke, L. A. et al. Calcium-activated SK potassium channels are key modulators of the pacemaker frequency in locus coeruleus neurons. Mol. Cell Neurosci. 88, 330-341. https://doi.org/10.1016/j.mcn.2018.03.002 (2018).

23. Wolfart, J., Neuhoff, H., Franz, O. \& Roeper, J. Differential expression of the small-conductance, calcium-activated potassium channel SK3 is critical for pacemaker control in dopaminergic midbrain neurons. J. Neurosci. 21, 3443-3456 (2001).

24. Murai, Y. \& Akaike, T. Orexins cause depolarization via nonselective cationic and $\mathrm{K}^{+}$channels in isolated locus coeruleus neurons. Neurosci. Res. 51, 55-65. https://doi.org/10.1016/j.neures.2004.09.005 (2005).

25. Pan, W. J., Osmanović, S. S. \& Shefner, S. A. Adenosine decreases action potential duration by modulation of A-current in rat locus coeruleus neurons. J. Neurosci. 14, 1114-1122 (1994).

26. Degli Esposti, M. Inhibitors of NADH-ubiquinone reductase: An overview. Biochim. Biophys. Acta 1364, 222-235. https://doi.org/ 10.1016/s0005-2728(98)00029-2 (1998).

27. Alam, M. \& Schmidt, W. J. Rotenone destroys dopaminergic neurons and induces parkinsonian symptoms in rats. Behav. Brain Res. 136, 317-324. https://doi.org/10.1016/s0166-4328(02)00180-8 (2002).

28. Höglinger, G. U. et al. Chronic systemic complex I inhibition induces a hypokinetic multisystem degeneration in rats. J. Neurochem. 84, 491-502. https://doi.org/10.1046/j.1471-4159.2003.01533.x (2003)

29. Inden, M. et al. Parkinsonian rotenone mouse model: reevaluation of long-term administration of rotenone in C57BL/6 mice. Biol. Pharm. Bull. 34, 92-96. https://doi.org/10.1248/bpb.34.92 (2011).

30. Li, N. et al. Mitochondrial complex I inhibitor rotenone induces apoptosis through enhancing mitochondrial reactive oxygen species production. J. Biol. Chem. 278, 8516-8525. https://doi.org/10.1074/jbc.M210432200 (2003).

31. Keating, D. J., Rychkov, G. Y., Giacomin, P. \& Roberts, M. L. Oxygen-sensing pathway for SK channels in the ovine adrenal medulla. Clin. Exp. Pharmacol. Physiol. 32, 882-887. https://doi.org/10.1111/j.1440-1681.2010.04279.x (2005).

32. Strøbaek, D. et al. Activation of human IK and SK Ca ${ }^{2+}$-activated $\mathrm{K}^{+}$channels by NS309 (6,7-dichloro-1H-indole-2,3-dione 3-oxime). Biochim. Biophys. Acta 1665, 1-5. https://doi.org/10.1016/j.bbamem.2004.07.006 (2004).

33. Dolga, A. M. \& Culmsee, C. Protective roles for potassium SK/KCa2 channels in microglia and neurons. Front. Pharmacol. 3, 196. https://doi.org/10.3389/fphar.2012.00196 (2012).

34. Dolga, A. M. et al. Subcellular expression and neuroprotective effects of SK channels in human dopaminergic neurons. Cell Death Dis. 5, e999. https://doi.org/10.1038/cddis.2013.530 (2014).

35. Dolga, A. M. et al. KCa2 channels activation prevents $\left[\mathrm{Ca}^{2+}\right]_{\mathrm{i}}$ deregulation and reduces neuronal death following glutamate toxicity and cerebral ischemia. Cell Death Dis. 2, e147. https://doi.org/10.1038/cddis.2011.30 (2011).

36. Allen, D. et al. SK2 channels are neuroprotective for ischemia-induced neuronal cell death. J. Cereb. Blood Flow Metab. 31 , 2302-2312. https://doi.org/10.1038/jcbfm.2011.90 (2011).

37. Freestone, P. S. et al. Acute action of rotenone on nigral dopaminergic neurons-Involvement of reactive oxygen species and disruption of Ca2+ homeostasis. Eur. J. Neurosci. 30, 1849-1859. https://doi.org/10.1111/j.1460-9568.2009.06990.x (2009).

38. Pan-Montojo, F. et al. Environmental toxins trigger PD-like progression via increased alpha-synuclein release from enteric neurons in mice. Sci. Rep. 2, 898. https://doi.org/10.1038/srep00898 (2012).

39. Deng, P. Y. et al. Voltage-independent SK-channel dysfunction causes neuronal hyperexcitability in the Hippocampus of Fmr1 knock-out mice. J. Neurosci. 39, 28-43. https://doi.org/10.1523/jneurosci.1593-18.2018 (2019).

40. Szatanik, M. et al. Behavioral effects of a deletion in Kcnn2, the gene encoding the SK2 subunit of small-conductance Ca ${ }^{2+}$-activated $\mathrm{K}^{+}$channels. Neurogenetics 9, 237-248. https://doi.org/10.1007/s10048-008-0136-2 (2008).

41. Mochel, F. et al. Variants in the SK2 channel gene (KCNN2) lead to dominant neurodevelopmental movement disorders. Brain https://doi.org/10.1093/brain/awaa346 (2020). 
42. Yee, A. G., Freestone, P. S., Bai, J. Z. \& Lipski, J. Paradoxical lower sensitivity of Locus Coeruleus than Substantia Nigra pars compacta neurons to acute actions of rotenone. Exp. Neurol. 287, 34-43. https://doi.org/10.1016/j.expneurol.2016.10.010 (2017).

43. Duda, J., Pötschke, C. \& Liss, B. Converging roles of ion channels, calcium, metabolic stress, and activity pattern of Substantia nigra dopaminergic neurons in health and Parkinson's disease. J. Neurochem. 139(Suppl 1), 156-178. https://doi.org/10.1111/jnc. 13572 (2016).

44. Espay, A. J., LeWitt, P. A. \& Kaufmann, H. Norepinephrine deficiency in Parkinson's disease: The case for noradrenergic enhancement. Mov. Disord. 29, 1710-1719. https://doi.org/10.1002/mds.26048 (2014).

45. Vermeiren, Y. \& De Deyn, P. P. Targeting the norepinephrinergic system in Parkinson's disease and related disorders: The locus coeruleus story. Neurochem. Int. 102, 22-32. https://doi.org/10.1016/j.neuint.2016.11.009 (2017).

46. McCall, J. G. et al. CRH engagement of the locus coeruleus noradrenergic system mediates stress-induced anxiety. Neuron 87, 605-620. https://doi.org/10.1016/j.neuron.2015.07.002 (2015).

47. Herrik, K. F. et al. CyPPA, a positive SK3/SK2 modulator, reduces activity of dopaminergic neurons, inhibits dopamine release, and counteracts hyperdopaminergic behaviors induced by methylphenidate. Front. Pharmacol. 3, 11. https://doi.org/10.3389/ fphar.2012.00011 (2012).

48. Johnson, S. M., Haxhiu, M. A. \& Richerson, G. B. GFP-expressing locus ceruleus neurons from Prp57 transgenic mice exhibit $\mathrm{CO}_{2} / \mathrm{H}^{+}$responses in primary cell culture. J. Appl. Physiol. 1985(105), 1301-1311. https://doi.org/10.1152/japplphysiol.90414.2008 (2008).

49. Ip, C. W. et al. AAV1/2-induced overexpression of A53T- $\alpha$-synuclein in the substantia nigra results in degeneration of the nigrostriatal system with Lewy-like pathology and motor impairment: a new mouse model for Parkinson's disease. Acta Neuropathol. Commun. 5, 11. https://doi.org/10.1186/s40478-017-0416-x (2017).

50. Koprich, J. B., Johnston, T. H., Reyes, M. G., Sun, X. \& Brotchie, J. M. Expression of human A53T alpha-synuclein in the rat substantia nigra using a novel AAV1/2 vector produces a rapidly evolving pathology with protein aggregation, dystrophic neurite architecture and nigrostriatal degeneration with potential to model the pathology of Parkinson's disease. Mol. Neurodegener. 5, 43. https://doi.org/10.1186/1750-1326-5-43 (2010).

51. Paxinos, G. \& Franklin, K. B. J. Paxinos and Franklin's the Mouse Brain in Stereotaxic Coordinates (eds. Paxinos, G. \& Franklin, K. B. J.) (Elsevier Acad. Press, 2013).

\title{
Acknowledgements
}

We are grateful to Oxana Nowak, Andrea Schubert, Sabine Anfimov and Christine Höft for excellent technical support.

\section{Author contributions}

L.A.M., W.H.O. and N.D. designed the study; L.A.M., A.S., B.L. and M.G. performed patch clamp recordings and analyzed data. A.M.D. and L.A.M. conducted primary cultures and calcium imaging in vitro experiments. M.A.K. and A.K.K. analyzed data; M.T.H., F.F.G. and B.L. performed stereotactic surgeries, conducted immunohistochemical staining and analyzed data. S.R. and L.A.M. performed and analyzed qPCR experiments. W.H.C. supports the stereological analyses. J.B.K. and J.M.B. provided the vectors. W.H.O., L.A.M. and N.D. supervised the project. L.A.M., M.A.K., M.T.H., F.F.G., W.H.O. and N.D. wrote the manuscript; W.H.O. and N.D. critically revised the manuscript. All authors read and approved the final manuscript.

\section{Funding}

Open Access funding enabled and organized by Projekt DEAL. W.H.O. is Hertie-Senior-Research Professor supported by the Charitable Hertie Foundation, Frankfurt/Main, Germany. L.A.M. has received a grant from the Behring-Röntgen Foundation. L.A.M. and W.H.O. have received a grant from the Research Funds of the University Clinic Giessen-Marburg, M.A.K. is the recipient of a Studienstiftung des Deutschen Volkes Fellowship and has received a grant from the DPG. B.L. is a fellow of DAAD. S.R. received a grant for this study from the Anneliese Pohl Stiftung (UKGM). A.D. is the recipient of a Rosalind Franklin Fellowship co-funded by European Union and University of Groningen.

\section{Competing interests}

The authors declare no competing interests.

\section{Additional information \\ Supplementary Information The online version contains supplementary material available at https://doi.org/ 10.1038/s41598-022-06832-1.}

Correspondence and requests for materials should be addressed to N.D.

Reprints and permissions information is available at www.nature.com/reprints.

Publisher's note Springer Nature remains neutral with regard to jurisdictional claims in published maps and institutional affiliations.

\begin{abstract}
Open Access This article is licensed under a Creative Commons Attribution 4.0 International License, which permits use, sharing, adaptation, distribution and reproduction in any medium or
format, as long as you give appropriate credit to the original author(s) and the source, provide a link to the Creative Commons licence, and indicate if changes were made. The images or other third party material in this article are included in the article's Creative Commons licence, unless indicated otherwise in a credit line to the material. If material is not included in the article's Creative Commons licence and your intended use is not permitted by statutory regulation or exceeds the permitted use, you will need to obtain permission directly from the copyright holder. To view a copy of this licence, visit http://creativecommons.org/licenses/by/4.0/.
\end{abstract}

(C) The Author(s) 2022 